\title{
Fluctuation-Based Super-Resolution Traction Force Microscopy
}

\author{
Aki Stubb, Romain F. Laine, Mitro Miihkinen, Hellyeh Hamidi, Camilo Guzmán, Ricardo Henriques, \\ Guillaume Jacquemet,* and Johanna Ivaska*
}

Cite This: Nano Lett. 2020, 20, 2230-2245

Read Online

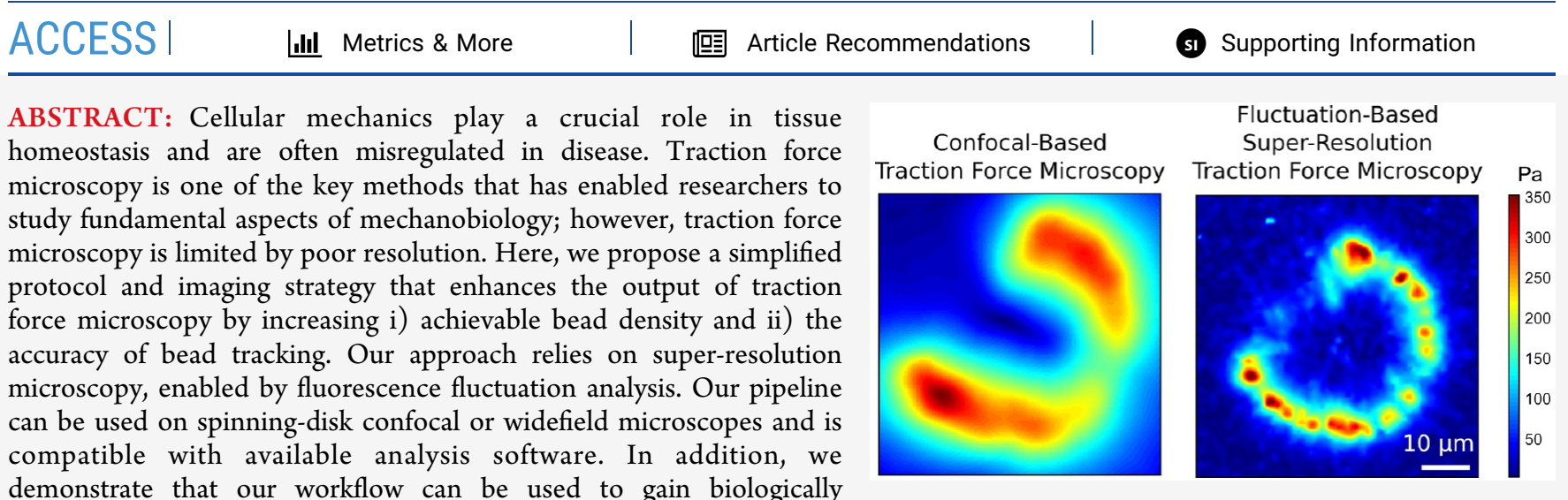
demonstrate that our workflow can be used to gain biologically relevant information and is suitable for fast long-term live measurement of traction forces even in light-sensitive cells. Finally, using fluctuation-based traction force microscopy, we observe that filopodia align to the force field generated by focal adhesions.

KEYWORDS: Fluctuation-based super-resolution microscopy, SACD, SRRF, traction force microscopy, live imaging, mechanobiology

ell adhesion to the extracellular matrix (ECM) is a

fundamental feature of multicellular life, and it is finely tuned during almost every cellular process including cell migration, cell proliferation, and cell fate. Cells are not passively attached to the ECM but instead constantly apply forces on ECM molecules and actively remodel their microenvironment. ${ }^{1}$ The major cellular structures responsible for transmitting these forces to the ECM are focal adhesions. ${ }^{1}$ These multiprotein signaling platforms also translate physical forces into intracellular biochemical signaling cascades. ${ }^{2}$ The ability of cells to apply mechanical forces on their environment is emerging as one of the key regulators of tissue patterning and morphogenesis, ${ }^{3,4}$ while dysregulation of this process is associated with diseases including aging, fibrosis, and cancer. $^{5-7}$ As our understanding of mechanobiology is rapidly unveiling promising novel therapeutic opportunities, ${ }^{8}$ the development of methods that can facilitate these investigations is of paramount importance.

While several strategies can be used to map and quantify the forces exerted by cells on their microenvironments, traction force microscopy (TFM) is one of the most convenient and widely used methods. ${ }^{9}$ To perform TFM, cells are allowed to adhere to a deformable material of defined stiffness, classically a polyacrylamide (PAA) gel, containing fluorescent beads. The forces exerted by cells on their substrate can then be monitored as a function of bead movement within the gel. As the stiffness and the elastic modulus of the gel are preestablished, bead displacement can then, using mathematical equations, be converted into a read-out of local forces. ${ }^{10}$
The sensitivity and accuracy of TFM are directly linked to the ability to detect and track moving beads within a thick gel and are generally limited to the detection of forces at the micron-scale. ${ }^{9,10}$ As focal adhesions can be small, ${ }^{11}$ there is a need to develop methods that can map cellular forces at high spatial resolution. For TFM, this can be achieved by (1) increasing the number of trackable beads ${ }^{12-14}$ and by (2) improving the computational algorithms used to map cellular forces. ${ }^{15}$ Multiple imaging and sample preparation strategies have been developed to increase the number of trackable beads in TFM experiments, each with unique strengths and shortcomings (Table 1). ${ }^{12-14}$ Improvements in TFM algorithms often require further biological assumptions ${ }^{16}$ as well as heavy computational processing power. ${ }^{15}$ Here we propose a simplified protocol that can resolve densely packed beads using software enabling super-resolution microscopy through fluorophore intensity fluctuation analysis. For simplicity, these algorithms are hereafter termed fluctuation-based superresolution (FBSR) imaging. In this study, we demonstrate that FBSR combined with TFM considerably enhances traction force outputs.

Received: October 3, 2019

Revised: March 2, 2020

Published: March 6, 2020 


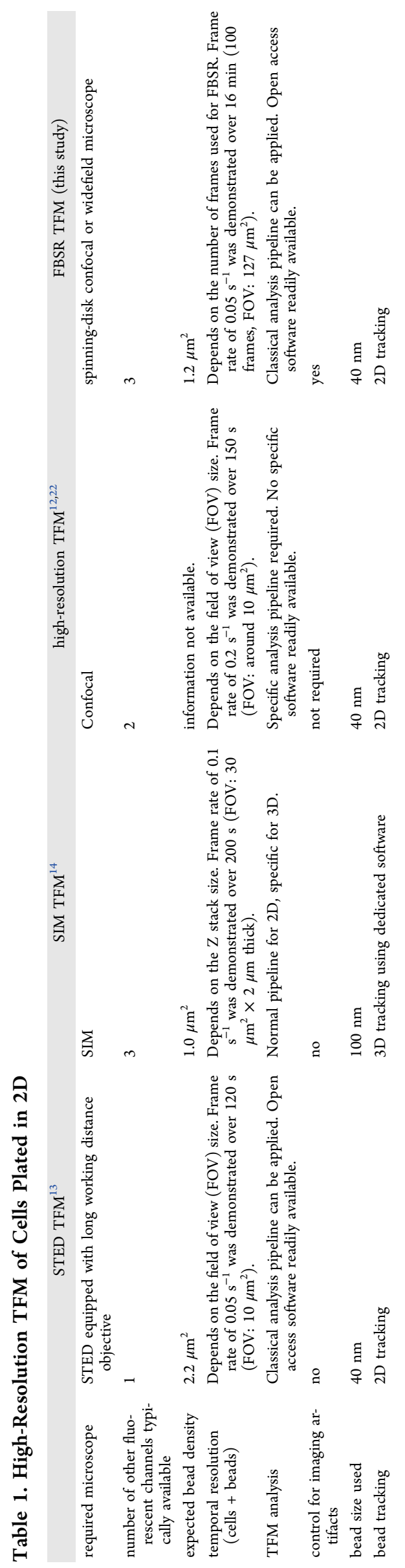

FBSR harnesses the intrinsic property of fluorophores that, when excited with continuous light, display random variation in intensity over time due to transitions between fluorescent and nonfluorescent states. ${ }^{17,18}$ After capturing these intensity oscillations (typically tens to hundreds of images), algorithms such as super-resolution optical fluctuation imaging (SOFI, ${ }^{19}$ ), super-resolution radial fluctuations $\left(\mathrm{SRRF}^{20}\right)$, or autocorrelation two-step deconvolution $(\mathrm{SACD})^{21}$ can be used to predict the location of fluorophores at improved resolution.

Increasing the Bead Density of TFM Gels Using Fluctuation-Based Microscopy. To increase the number of trackable beads in our TFM experiments, we decided to employ FBSR (Figure 1a) as it has several advantages over other SR modalities (Table 1). Of note, FBSR is easy to implement, is compatible with most pre-existing microscopes including spinning-disk confocal and widefield systems, ${ }^{20}$ and is only mildly phototoxic with improved resolution being achieved using illumination intensities typical for conventional fluorescence imaging. ${ }^{23}$ Classically, when performing TFM experiments, $200 \mathrm{~nm}$ fluorescent beads are embedded throughout the PAA gel. ${ }^{10}$ This can result in substantial outof-focus light, which limits the use of widefield microscopes for TFM. To increase the number of trackable beads in our TFM experiments and enable better quantification of cellular forces, we used gels containing densely packed $40 \mathrm{~nm}$ fluorescent beads.

To validate that FBSR can improve the detection of $40 \mathrm{~nm}$ beads, we performed simulations with known and increasing bead densities (see Materials and Methods for details; Supplementary Figure $1 \mathrm{a}-\mathrm{d}$ ). These simulations show that, at low bead densities, accurate bead numbers can be recovered from both widefield and FBSR images with FBSR processing clearly improving the quality and resolution of the final images (Supplementary Figure 1a,b). However, at higher bead densities (over 1 bead per square micrometer), FBSR processing allowed a higher recovery of bead numbers compared to the widefield images (Supplementary Figure $1 \mathrm{a}, \mathrm{b})$. To assess the improvement in bead trackability enabled by the detection of higher bead density using FBSR processing, a realistic displacement field was applied to our simulated data (see Materials and Methods for details; Supplementary Figure 1c). The bead displacement maps generated using FBSR imaging demonstrated that while the overall displacement field was apparent at low bead densities, fine details could only be retrieved at high bead densities (Supplementary Figure 1c,d). Altogether, our simulations demonstrate that FBSR processing allows for the detection of higher bead densities, which leads to increased trackability of the beads after image reconstruction and in turn to improved recovery of spatial details in the force map.

To optimize TFM gels for FBSR, and inspired by previous work, ${ }^{13,15,24-26}$ we optimized a simplified gel casting protocol where the $40 \mathrm{~nm}$ beads are embedded only on the topmost layer of the gel (Supplementary Figure 2a,b). This was achieved by precoating the top coverslip, used to flatten the gel solution prior to casting, with the beads instead of mixing the beads within the gel solution itself (Supplementary Figure 2a). Importantly, using the FBSR algorithms LiveSRRF and SACD and our optimized protocol, we were able to improve the detection of $40 \mathrm{~nm}$ beads located on top of the TFM gel using both spinning-disk confocal and widefield microscopes (Figure 1b). To ensure that as few artefacts as possible were introduced during the FBSR reconstruction process, the 


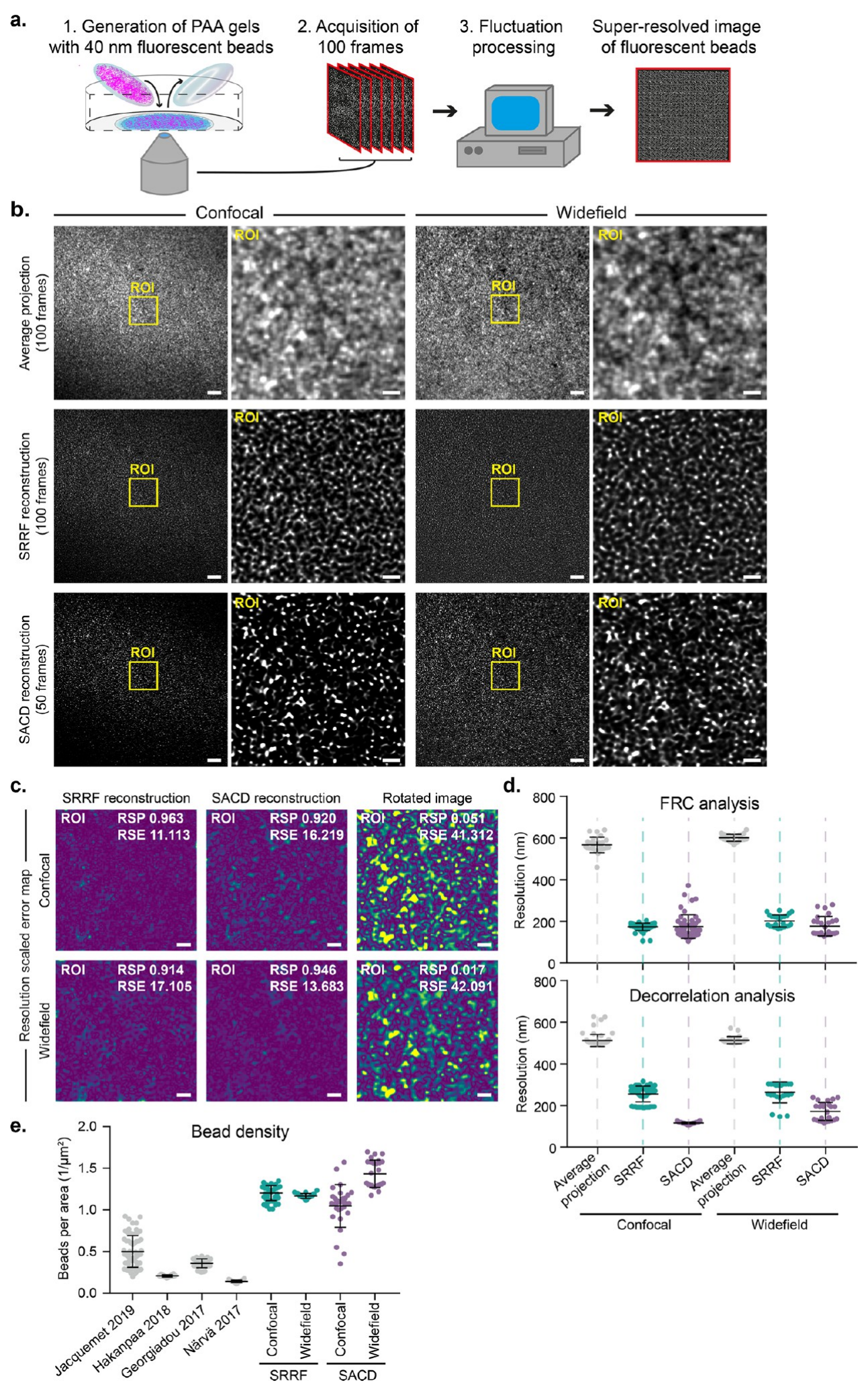

Figure 1. FBSR processing enhances bead recognition in TFM specific PAA gels. (a) Cartoon illustrating the key steps used to produce TFM gels for FBSR imaging. (1) TFM gel where $40 \mathrm{~nm}$ fluorescent beads are embedded only on the topmost layer of the gel is generated. (2) TFM gels are then imaged using a spinning-disk confocal or widefield microscope. To allow for FBSR processing, each field of view is imaged 100 times. (3) SR images are then generated using available FBSR algorithms such as LiveSRRF or SACD. (b) TFM gel prepared using our improved protocol (40 $\mathrm{nm}$ beads embedded only at the top) was imaged using spinning-disk confocal or widefield modes. To allow for FBSR, 50 (SACD) or 100 (SRRF) frames were recorded. Average projections, LiveSRRF and SACD images are displayed. For each condition, the yellow square highlights a region of interest (ROI) that is magnified. All images are from the same field of view. Scale bar: (main) $10 \mu \mathrm{m}$; (inset) $2 \mu \mathrm{m}$. (c) Resolution scaled error maps of the LiveSRRF and SACD images displayed in b (ROI only) generated with NanoJ-SQUIRREL. Maps are color-coded to visualize areas of low (purple) and high error (yellow). As a control, the same analysis was performed using a reference frame that was rotated $90^{\circ}$. Scale bars $2 \mu \mathrm{m}$. 
Figure 1. continued

(d) Estimation of the resolution of bead images before and after FBSR processing using Fourier ring correlation (FRC) or decorrelation analysis (see Materials and Methods for details). Results are displayed as dot plots (average projections confocal, $n=26, n=63$; LiveSRRF confocal, $n=53$, $n=84$; SACD confocal, $n=53, n=56$; average projections widefield, $n=24, n=26$; LiveSRRF widefield, $n=26, n=24$; SACD widefield, $n=26, n$ =26). (e) Graph showing bead densities (beads per square micrometer) measured from multiple published TFM data sets ${ }^{28-31}$ and from the TFM gels (improved protocol described here) imaged using either spinning-disk confocal or widefield followed by FBSR processing using LiveSRRF or SACD.

image quality was assessed using NanoJ SQUIRREL ${ }^{27}$ and the resolution scaled Pearson's correlation (RSP) and resolution scaled error (RSE) parameters were calculated by the software (Figure 1c). In addition to these parameters, when choosing the reconstruction settings, the amount of beads detected and the absence of patterning in the final image were also taken into consideration (Supplementary Figure 2c,d). FBSR processing led to a 2-3-fold improvement in the resolution of bead images as measured by Fourier ring correlation and decorrelation analyses (Figure 1d).

Prior to FBSR, our confocal-based TFM analyses have yielded between 0.2 to 0.5 trackable beads per square micrometer $^{28-31}$ (Figure 1e), in agreement with values reported by others. ${ }^{13}$ Here, by taking advantage of the densely packed $40 \mathrm{~nm}$ bead layer gels and by implementing FBSR, and conservative reconstruction parameters, we were able to substantially increase the number of trackable beads to 1.2 beads per square micrometer (Figure 1e). This is a modest improvement over a protocol using structured illumination microscopy $^{14}$ ( 1 bead per square micrometer) but remains inferior to another protocol based on STED imaging within small fields of view ( 2.2 beads per square micrometer) (Table 1). ${ }^{13}$ Interestingly, FBSR performed especially well when images were acquired using widefield microscopy as the final SR images were more homogeneous (Figure $1 \mathrm{~b}$ ). When the images were acquired using spinning-disk confocal, the corners of the field of view were often off focus due to uneven/ wrinkled gels resulting in much lower bead density in these areas. In particular, spinning-disk confocal images reconstructed by SACD appear to be especially sensitive to out of focus light, arisen from uneven gels, leading to more variable bead density than the other imaging modalities (Figure $1 \mathrm{~b}$ ). However, when imaging cellular structures (e.g., cytoskeleton or focal adhesions) on TFM gels, spinning-disk confocal imaging is likely to outperform widefield imaging and may be favored despite generating less homogeneous bead images.

Implementation of Fluctuation-Based Traction Force Microscopy. In a typical TFM experiment, beads are imaged before (pre) and after (post) removing cells, the pre and post images are then aligned, and the beads are detected and tracked in both images. ${ }^{10}$ From the tracking data, bead displacement maps and force maps can be generated using available TFM software. ${ }^{15,32}$ FBSR-TFM follows the same workflow with the addition of image reconstruction prior to the pre and post image alignment (Figure 2a).

To assess the improvement generated by FBSR-TFM over classically used confocal-based TFM, cells were plated on gels containing both $200 \mathrm{~nm}$ beads (distributed throughout the gel, classic protocol) and $40 \mathrm{~nm}$ beads (distributed only at the top of the gel, new protocol described here) (Figure $2 b, c$ and Supplementary Figures $2 \mathrm{~b}$ and 3 ). This strategy enabled us to measure and visualize traction forces using both methodologies within the same field of view (Figure 2). Using spinning-disk confocal imaging of the $200 \mathrm{~nm}$ beads (classic confocal TFM) or of the $40 \mathrm{~nm}$ beads, we were able to track 8253 beads and 11328 beads, respectively (full field of view, Supplementary Figure 3). In contrast, FBSR imaging of the $40 \mathrm{~nm}$ beads, yielded 20799 (LiveSRRF processing using 100 frames) and 22908 trackable beads (SACD processing using 50 frames) within the same field of view. The spinning-disk confocal-based TFM generated displacement and traction force maps that closely recapitulated the shape of the cell (Figure 2c). However, at this resolution, areas corresponding to cell-ECM contacts such as focal adhesions could not be pinpointed, and results were not substantially improved when using the spinning-disk confocal images of the $40 \mathrm{~nm}$ beads (Figure 2c). Strikingly, applying the same TFM pipeline to the FBSR images of the $40 \mathrm{~nm}$ beads (regardless of the FBSR and the bead tracking methods used) drastically improved the resolution of the displacement and force maps (Supplementary Figures 3 and 4). In particular, defined regions of high forces were specifically detected at the cell perimeter, which could correspond to focal adhesions (Figure $2 \mathrm{c}$ and Supplementary Figure 4). In addition, due to enhanced bead tracking, we could better segregate cellular regions corresponding to weaker forces. While the final force maps are affected by the algorithm/mathematical framework used to perform force reconstruction, ${ }^{15}$ the bead displacement maps are a direct reflection of the amount of beads used as well as the quality of bead tracking. ${ }^{9,12}$ In particular, errors in displacement measurements caused by a lack of accuracy in the tracking routines strongly affect the resolution of TFM. Notably, in the case of the spinning-disk confocal TFM, large beads are only tracked over subpixel movements, which is likely to lead to tracking inaccuracies (Figure 2c Supplementary Figure 3). In contrast, in the case of FBSR-TFM the tracking accuracy is likely to be improved as (1) the beads are smaller and (2) they are now tracked over several pixels (due to the smaller pixel size of the FBSR images). Overall, we believe that FBSR improves the TFM outputs by both increasing the bead density (more data points) and by refining the accuracy of bead tracking.

Versatility of Fluctuation-Based TFM. One of the advantages of FBSR is that it can easily accommodate multicolour imaging. To demonstrate this capability, we set out to measure forces in cells endogenously tagged for paxillin, a marker of focal adhesions. In this case, FBSR not only enhanced bead tracking and identification but also the resolution in images of paxillin-positive focal adhesions (Figure 3a, Supplementary Figure 5a). Importantly, this easy multicolour imaging capability combined with enhanced image quality enabled us to confirm that the observed regions of higher force correlate with the localization of cell-ECM adhesions (Figure 3a,b).

To demonstrate that FBSR is compatible with multiple existing TFM pipelines, we compared the displacement and force maps generated by two freely available software (MATLAB $^{15}$ or ImageJ-based software; ${ }^{32}$ see Materials and 
a.

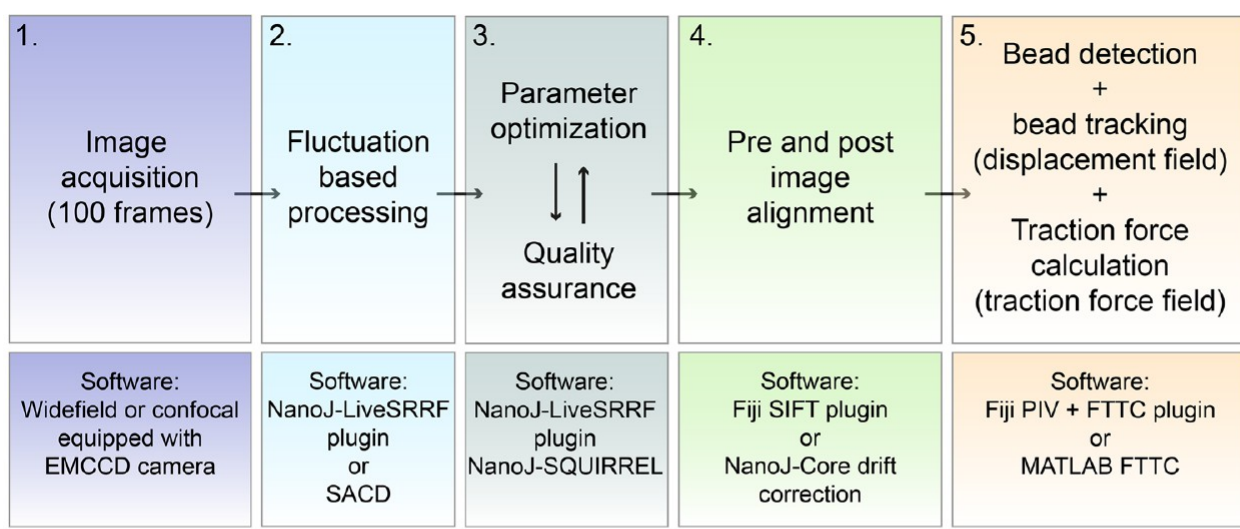

b.

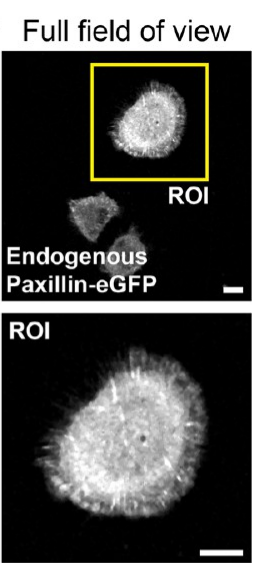

c.
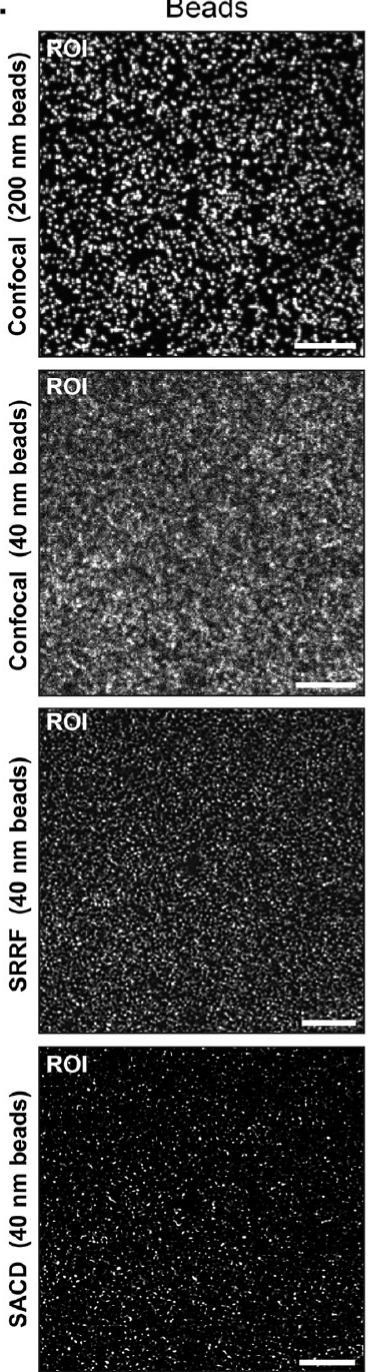

Beads + displacement
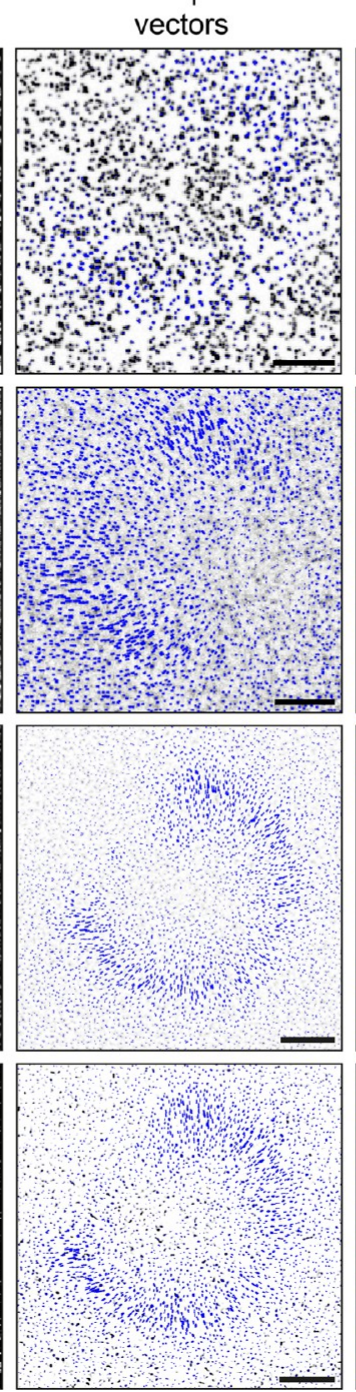
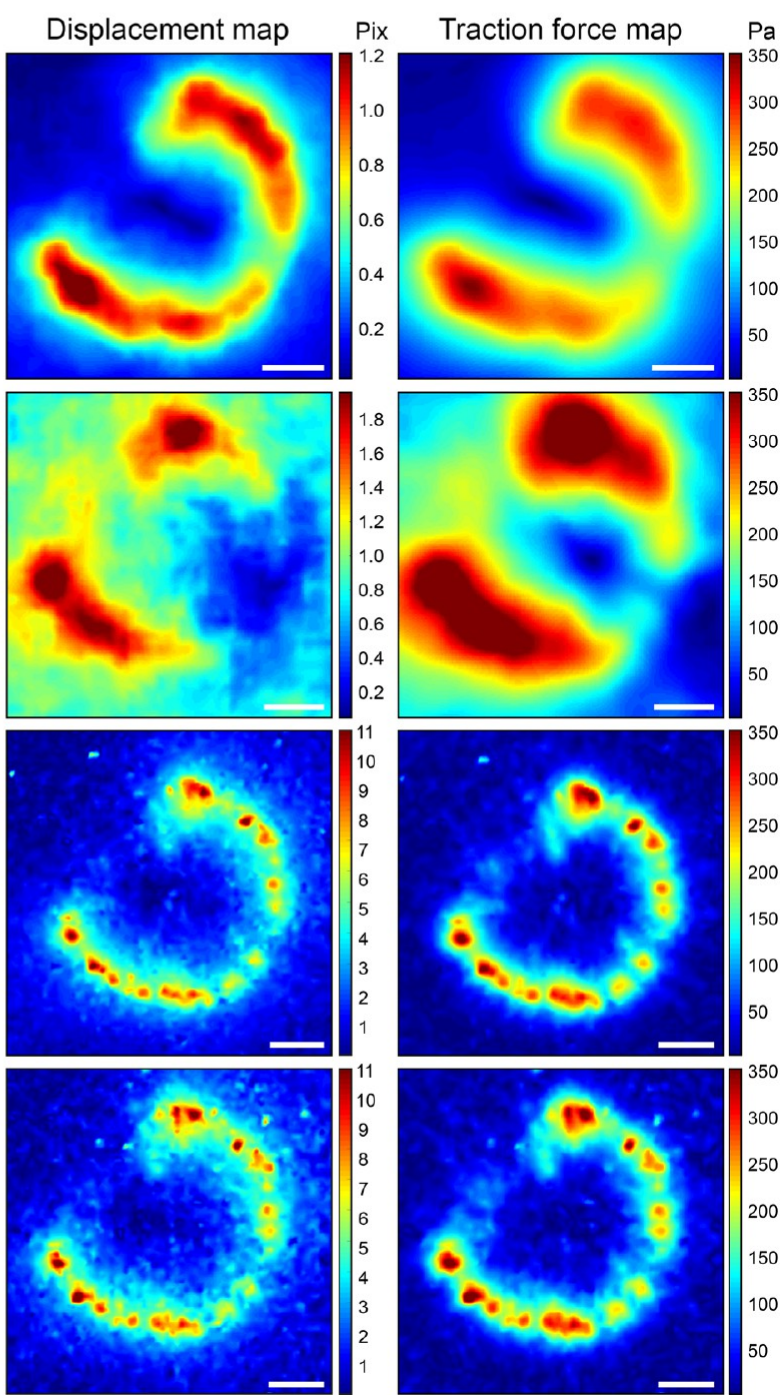

Figure 2. Implementation of FBSR for TFM. (a) Schematic pipeline of a TFM experiment that includes FBSR and image quality control. The software needed to complete each step are listed. $(b, c)$ To assess the improvement generated by FBSR-TFM over the classically used confocalbased TFM, U2OS cells expressing endogenously tagged paxillin were plated on $9.6 \mathrm{kPa}$ gels containing both 40 and $200 \mathrm{~nm}$ beads and TFM analyses were performed (as in panel a) on the ROI (yellow square, b). Spinning-disk confocal images of 200 and $40 \mathrm{~nm}$ beads and FBSR images of the $40 \mathrm{~nm}$ beads (LiveSRRF; SACD) were used for TFM analysis using a MATLAB-based software. ${ }^{15}$ For each method, images of beads alone and beads (black) + displacement vectors (blue arrows, length scaled up by 2) and maps of bead displacement and traction force are displayed (c). The magnitudes of bead displacement and traction force are color-coded as indicated. Scale bars $10 \mu \mathrm{m}$. Analyses of the full field of view from panel $\mathrm{b}$ can be found in Supplementary Figure 3. Bead tracking was performed here using cross-correlation within the search window. The same analysis performed using PIV can be found in Supplementary Figure 4. 
a.

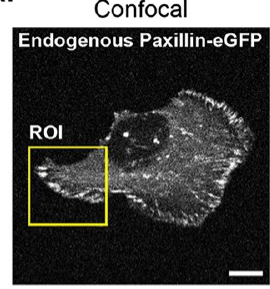

SRRF

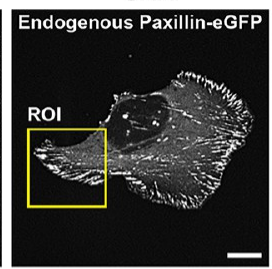

Displacement map
Confocal

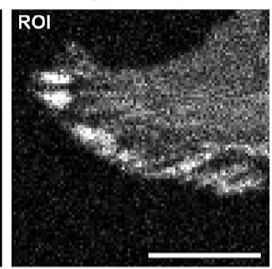

Resolution scaled

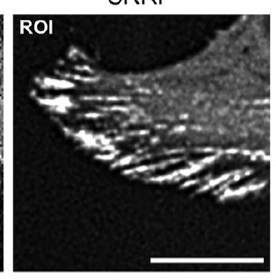

Traction force map

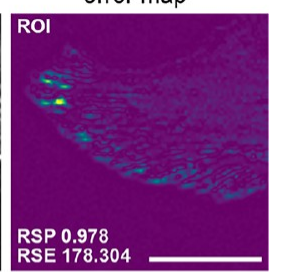

\section{$\mathrm{Pa}$}

b.

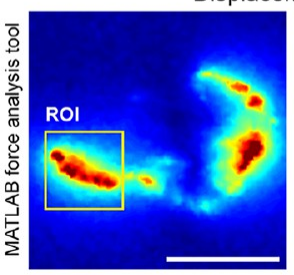

c.
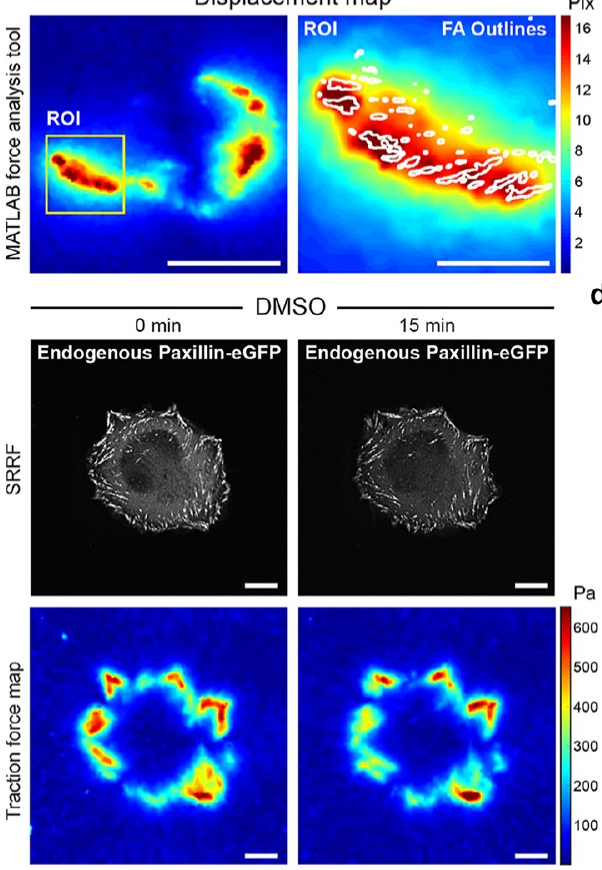

e.

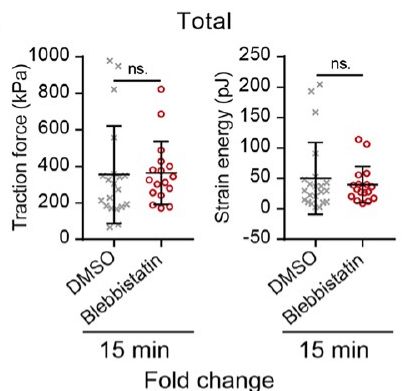

g.

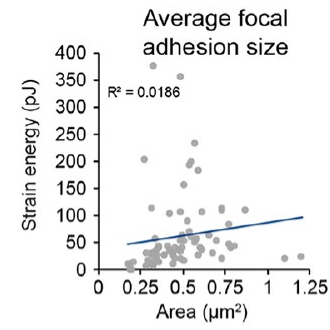

d.
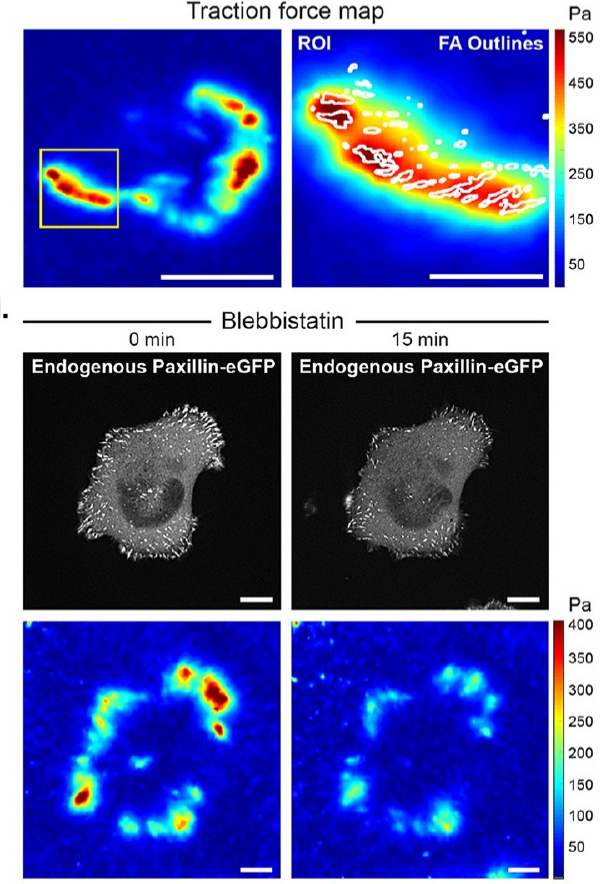
. 


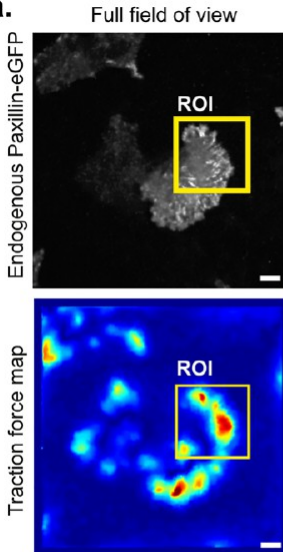

b.
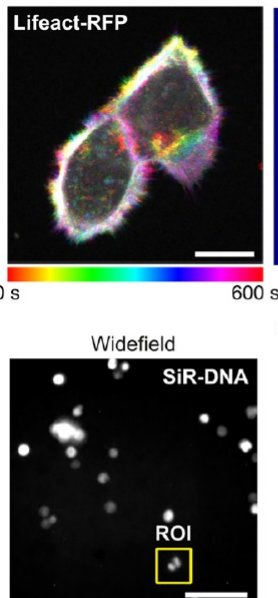

ROI SiR-DNA

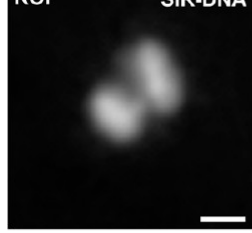

e.
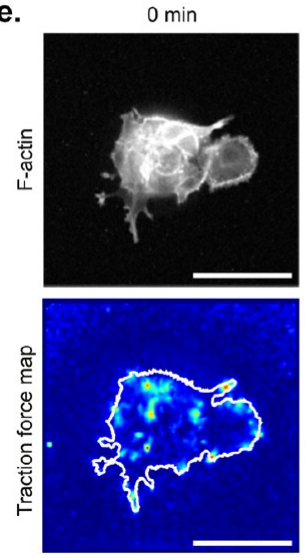

0 min
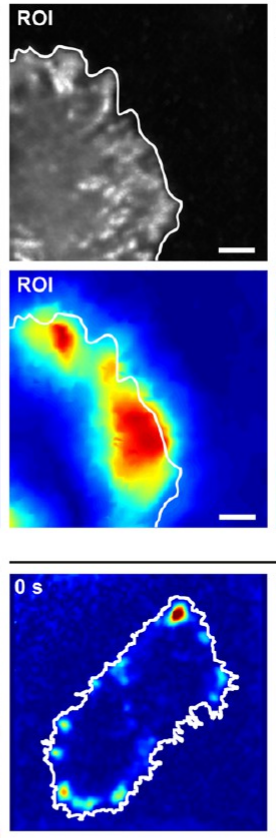

d.
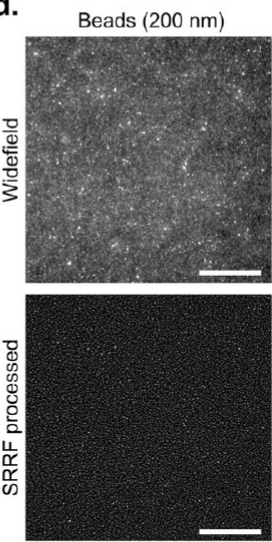

$25 \mathrm{~min}$
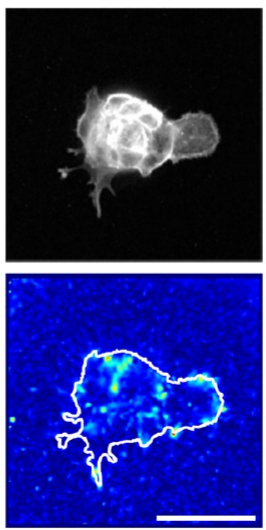
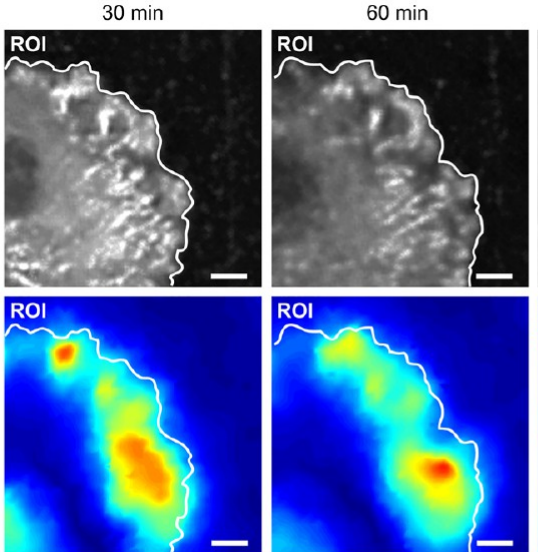

$90 \mathrm{~min}$

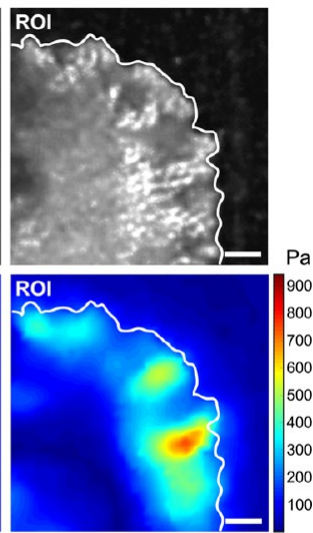

Traction force map
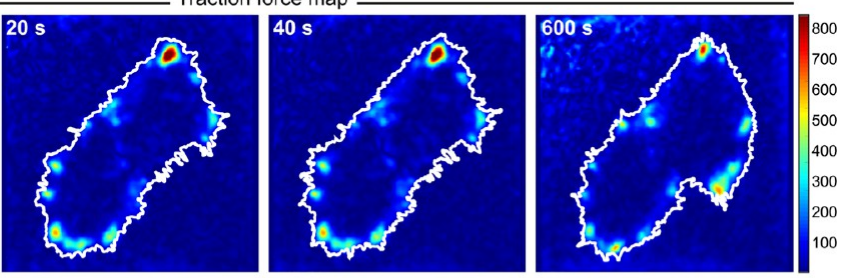

Traction force map
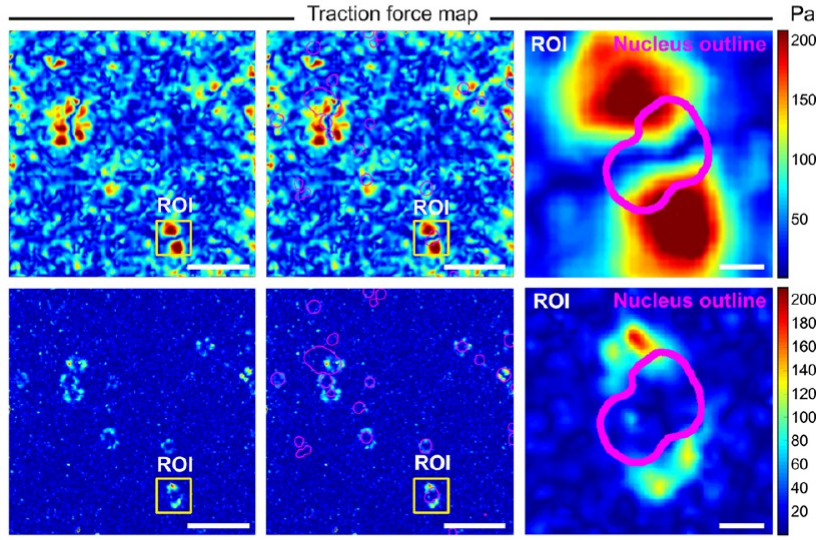

$60 \min$
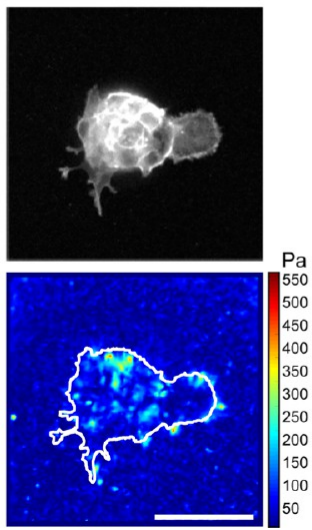

f.

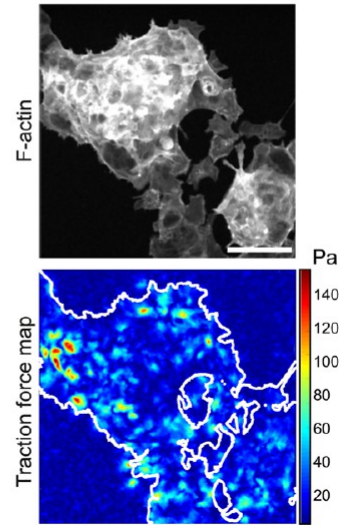

Figure 4. Live cell imaging and large field of view FBSR-TFM. (a) U-251 glioma cells expressing endogenously tagged paxillin were plated on 9.6 kPa TFM gels containing $40 \mathrm{~nm}$ beads. Cells were imaged live, every 5 min and FBSR-TFM was performed (spinning-disk confocal imaging, LiveSRRF processing and TFM analysis using MATLAB). The paxillin channel was denoised using the Noise2VOID algorithm. ${ }^{34}$ A representative field of view is displayed for the paxillin channel as well as the matching traction force map. The yellow square highlights a ROI that is magnified and displayed for several time points. The full movie is provided as Supporting Information (Video 1). White line depicts the leading edge of the cell. Scale bar: (main) $10 \mu \mathrm{m}$; (inset) $5 \mu \mathrm{m}$. (b) DCIS.COM lifeact-RFP cells were plated on $9.6 \mathrm{kPa}$ TFM gels containing $40 \mathrm{~nm}$ beads. Cells were imaged live, every $20 \mathrm{~s}$ ( $80 \mathrm{~ms}$ exposure time per frame) over $16 \mathrm{~min}$ and FBSR-TFM was performed (spinning-disk confocal imaging, Live-SRRF processing and TFM analysis using MATLAB). A time projection of the lifeact channel and matching traction force maps for several time points are displayed. The full movie is provided as Supporting Information (Video 2). White line depicts the leading edge of the cell. Scale bar: $25 \mu \mathrm{m}$. (c, d) 
Figure 4. continued

U2OS cells were plated on $2.6 \mathrm{kPa}$ TFM gels containing $200 \mathrm{~nm}$ beads (classic protocol), (c) treated with SiR-DNA to label nuclei, and imaged for FBSR TFM using a widefield microscope $\left(20 \times\right.$ air objective). (c) The SiR-DNA images were denoised using the Noise2VOID algorithm ${ }^{34}$. Both widefield and FBSR images (LiveSRRF) were used to perform TFM analyses (MATLAB software). (d) Images of the beads and the matching traction force maps are displayed. The outline of the nucleus is overlaid in magenta. Yellow squares highlight ROI that are magnified. Scale bar: (main) $100 \mu \mathrm{m}$; (inset) $10 \mu \mathrm{m}$. (e, f) Spheroids were generated from DCIS.COM lifeact-RFP cells kept in suspension for 7 days. Spheroids were then seeded on top of $9.6 \mathrm{kPa}$ TFM gels containing $200 \mathrm{~nm}$ beads (classic protocol) for $24 \mathrm{~h}$ before being imaged using a confocal microscope (20X air objective). Spheroids were imaged live, every $5 \mathrm{~min}$ and FBSR-TFM was performed (Live-SRRF processing and TFM analysis using MATLAB). (e) Several time points of a representative field of view are displayed for the lifeact channel as well as the matching traction force maps. (f) A single time point of a larger cell cluster is displayed. White lines depict the outline of the cell clusters. Scale bars: $100 \mu \mathrm{m}$.

Methods for details). Regardless of the software used, the displacement and force maps matched well to the outline of the focal adhesions (Supplementary Figure $5 b-e$ ). Furthermore, the total amount of forces measured using these two software showed a remarkable correlation (Supplementary Figure 5e).

Biologically Relevant Applications of FluctuationBased TFM. Next, we sought to demonstrate that our improved TFM pipeline could be used to answer biologically relevant questions. In particular, TFM is very commonly used to assess how a protein or a drug treatment influences the ability of cells to exert forces on their environment. ${ }^{28,29,31}$ For this purpose, we aimed to cause a mild perturbation to simulate a plausible biological response and treated cells with either DMSO or the myosin II inhibitor blebbistatin for 15 min (Figure 3c,d). FBSR imaging of focal adhesions and FBSR TFM measurements were performed before and after the treatments to allow the quantification of force changes in each cell. Importantly, 15 min of treatment with blebbistatin was not sufficient to trigger the collapse of focal adhesions (Figure $3 \mathrm{c}, \mathrm{d})$. While blebbistatin treatment triggered a notable decrease in the traction forces exerted by cells (Figure $3 c, d$ ), this effect was masked by the high variability in both DMSO and blebbistatin-treated cell populations when comparing the 15 min time point only (Figure $3 \mathrm{e}$ ). Therefore, we directly compared cells before (time point 0) and after treatments (time point $15 \mathrm{~min}$ ) and calculated the fold change in traction force and strain energy (SE) for each condition (DMSO and blebbistatin). We found that blebbistatin treatment significantly decreased both traction forces and SE (Figure 3f), in line with our traction force maps. These results indicate that the negative effect of blebbistatin on cellular forces, usually associated with a dramatic loss in focal adhesions, occurs, and can be detected by FBSR-TFM, at earlier stages preceding focal adhesion disassembly. In addition, our FBSR-TFM analysis pipeline highlights the value of performing TFM prior to and after a perturbation on the same cell to remove cell-to-cell variability ${ }^{33}$ and thus to more accurately detect changes in cellular forces. Interestingly, in this data set, the SE exerted by an individual cell did not correlate with individual focal adhesion properties such as "average focal adhesion size" or "paxillin intensity" at focal adhesions. Instead, forces generated by cells correlated well with cell-wide parameters such as "cell area" and "total area covered by focal adhesions" (Figure 3g).

Live-Cell FBSR TFM. FBSR is only mildly phototoxic, an important property for extended live-cell imaging. ${ }^{23}$ Therefore, we next sought to assess if FBSR would be suitable for extended live TFM experiments. Glioma cells with endogenously tagged paxillin were imaged every $5 \mathrm{~min}$, over a 100 min time period and FBSR TFM measurements were performed (Figure 4a, Video 1 and Supplementary Figure 6a). In this experiment, the signal-to-noise ratio of endogenous paxillin was improved using a recent denoising approach based on convolutional neural network. ${ }^{34}$ Using these images, modulation of forces could clearly be observed, at high resolution, as cells protruded (Figure 4a, Video 1, Supplementary Figure 6a). Glioma cells imaged using this strategy were not visibly disturbed by the imaging. In addition, the same strategy could be used to perform extended live TFM imaging of human-induced pluripotent stem cells, which, in our experience, are very sensitive to phototoxicity (Supplementary Figure 6b).

The temporal resolution of FBSR TFM depends on (1) the number of frames used for the reconstruction, (2) the exposure time used for the acquisition of individual images, and (3) the number of channels to image. We could perform TFM measurement at maximal resolution (100 frames, $80 \mathrm{~ms}$ exposure time), in breast cancer cells, every $20 \mathrm{~s}$ over $16 \mathrm{~min}$ (Figure $4 \mathrm{~b}$ and Video 2). At this speed, force fluctuations in defined regions of high force were clearly visible (Video 2), resembling the tugging behavior of focal adhesions described by others. ${ }^{22}$ If faster acquisition speeds are required, the parameters listed above can be carefully tuned, but decreasing them too much may result in lowering the final image resolution (Supplementary Figure 7). Altogether, our data demonstrate that FBSR-TFM is suitable for fast long-term live TFM imaging.

Large Field of View FBSR TFM. Next, we tested the capability of FBSR to improve bead detection over very large fields of view $(399 \mu \mathrm{m} \times 399 \mu \mathrm{m})$ using low magnification objectives. In this case, single cells were plated on gels containing $200 \mathrm{~nm}$ fluorescent beads and imaged using a $20 \times$ air objective (Figure 4c,d). TFM analyses were performed on both the widefield and FBSR images (Figure 4c,d). In the case of single cells, the force maps generated from widefield images were of poor quality and relatively noisy with forces being detected in cell-free areas (Figure 4c,d). In contrast, FBSR processing of the same field of view drastically improved traction force maps and areas of high force closely matched the outline of individual cells (Figure $4 \mathrm{c}, \mathrm{d}$ ). To assess if this variant of FBSR-TFM is also capable of measuring forces exerted by cell clusters, organoids generated from breast cancer cells were plated on fibronectin-coated gels containing $200 \mathrm{~nm}$ fluorescent beads and imaged live every $5 \mathrm{~min}$ over $1 \mathrm{~h}$ (Figure $4 \mathrm{e}, \mathrm{f}$ ). In this data set, we were able to detect forces that match closely the shape of the cell clusters as well as to pinpoint high forces within small and dynamics protrusions (Figure 4e,f). We believe that this variant of FBSR-TFM could prove useful to improve force measurements in migrating cell monolayers or to perform high throughput TFM screens. ${ }^{35,36}$ 
a.

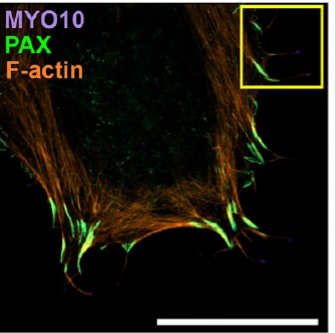

b.
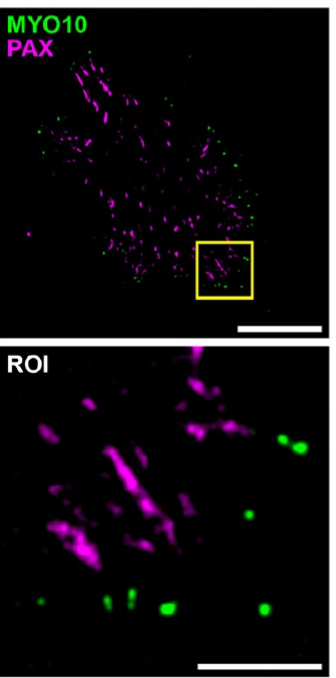

d.

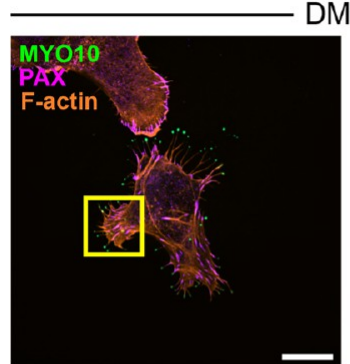

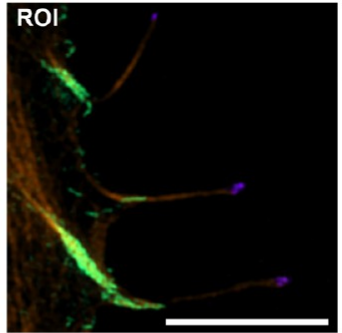

+ Displacement vectors

\section{MYO10}
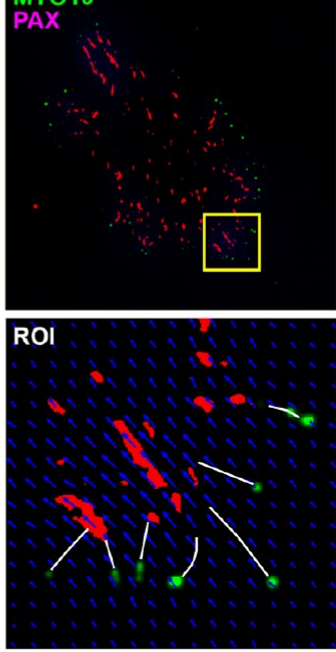

MSO

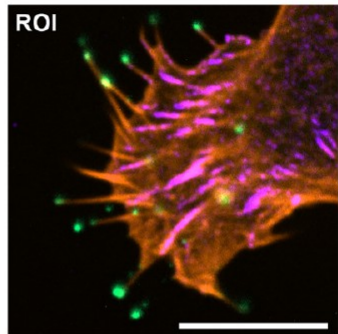

- Connected to a FA

$\checkmark$ Not connected to a FA

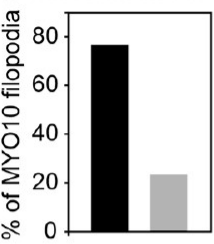

Traction force map

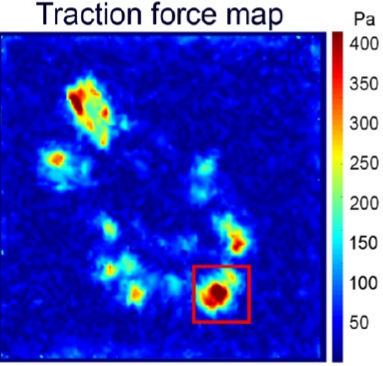

c.
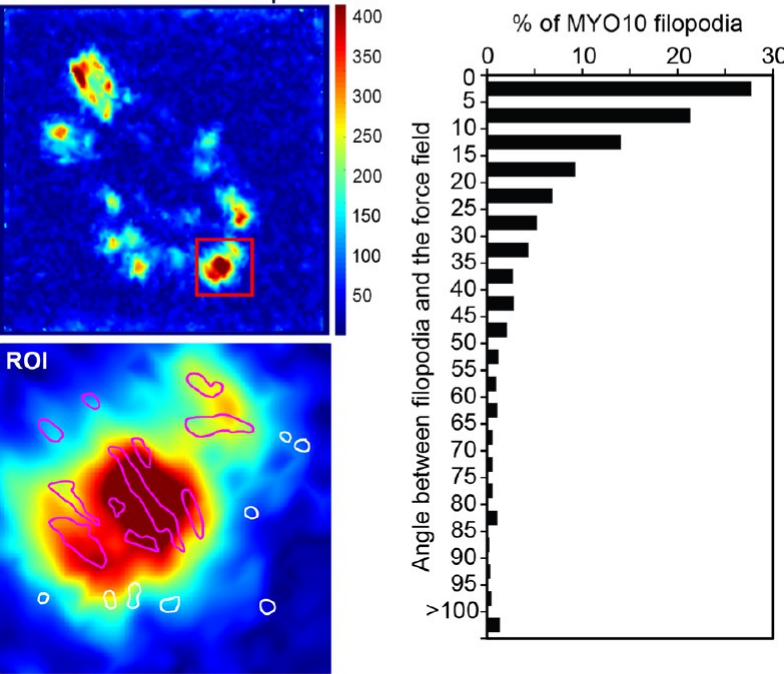

e.
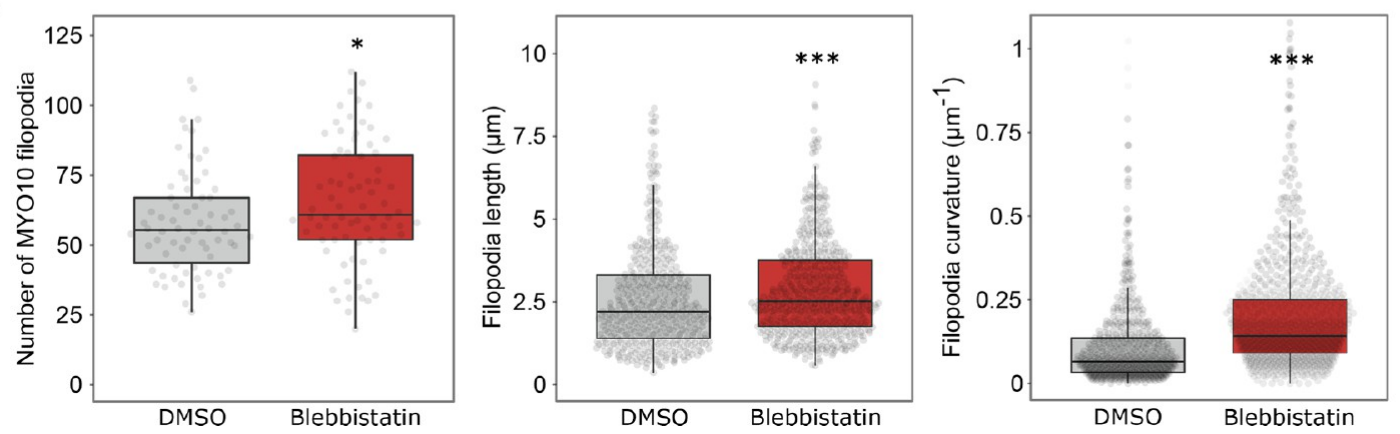

Figure 5. Relationship between filopodia adhesions and focal adhesions. (a) U2OS cells transiently expressing Paxillin-GFP and Myosin-X-mScarlet were plated on fibronectin-coated glass bottom dishes, fixed, stained for F-actin, and imaged using structured illumination microscopy. A representative field of view is displayed. The yellow square highlights a ROI that is magnified. Scale bar: (main) $20 \mu \mathrm{m}$; (inset) $5 \mu \mathrm{m}$. The percentage of filopodia directly connected to a paxillin-positive focal adhesion (FA) was quantified and the results are displayed as a bar chart. (b, c) U2OS cells transiently expressing Paxillin-mKate2 and Myosin-X-GFP were plated on $9.6 \mathrm{kPa}$ TFM gels containing $40 \mathrm{~nm}$ beads. Cells were imaged using a spinning-disk confocal and FBSR-TFM was performed (Live-SRRF processing and TFM analysis using MATLAB). In this data set, the beads, myosin-X (MYO10) and paxillin were imaged for FBSR processing. A representative field of view is displayed for the paxillin and MYO10 channels with and without the displacement vectors (blue arrows, length scaled up by 2) as well as the matching traction force map. The yellow square highlights a ROI that is magnified. The white lines in the displacement vectors map indicate the filopodia shafts (visible as very low intensity in the MYO10 channel). White circles in the traction map depict the location of filopodia tips. (b) Scale bar: (main) $20 \mu \mathrm{m}$; (inset) $5 \mu \mathrm{m}$. The alignment of filopodia tips to the force field was then measured using ImageJ. (c) The results are displayed as a frequency bar chart $(n=1022$ filopodia). (d, e) U2OS cells transiently expressing Myosin-X-GFP were plated on fibronectin-coated glass bottom dishes for $1 \mathrm{~h}$ before being 
Figure 5. continued

treated with either DMSO or $10 \mu \mathrm{M}$ blebbistatin for $1 \mathrm{~h}$. Cells were fixed and stained for paxillin and actin before being imaged using a spinningdisk confocal. A representative field of view is displayed. The yellow square highlights a ROI that is magnified. (d) Scale bar: (main) $20 \mu \mathrm{m}$; (inset) $10 \mu \mathrm{m}$. For each condition, the number of MYO10-positive filopodia per cell (DMSO, $n=70$ cells; blebbistatin, $n=77$ cells; $* p$ value $=0.049$ ), their length (DMSO, $n>446$ filopodia; blebbistatin, $n=945$ filopodia; $* * * p$ value $<0.001$ ), and their curvature (DMSO, $n=640$ filopodia; blebbistatin, $n=945$ filopodia; $* * * p$ value $<0.001$ ) were quantified. (e) $P$-values were determined using a randomization test.

Investigating the Mechanical Relationship between Focal Adhesions and Filopodia. Filopodia are small and dynamic finger-like actin-rich protrusions and are often the very first point of contact between a cell and its immediate surroundings. ${ }^{37} \mathrm{We}$ and others have previously described that integrin-mediated mechanosensitive adhesions form at filopodia tips and that these filopodia adhesions can mature into focal adhesion. ${ }^{28,38}$ Interestingly, the formation of filopodia adhesions has been reported to require cellular contractility. ${ }^{39}$ In U2OS cells expressing MYO10-GFP (to visualize and induce filopodia formation) we found that $80 \%$ of filopodia are directly connected to a paxillin-positive focal adhesion (Figure $5 a)$. To investigate the mechanical interplay between filopodia adhesions and focal adhesions, we took advantage of the increased resolution and multicolour capability of FBSR-TFM. U2OS cells expressing MYO10-GFP and paxillin-RFP were plated on TFM gels and FBSR-TFM measurements were performed (Figure 5b). Importantly, all detected cellular forces could be mapped back to focal adhesions and the forces generated by filopodia adhesions appeared to be negligible. Further careful analysis of the force maps revealed that filopodia tend to align to the force field generated at focal adhesions (Figure $5 \mathrm{~b}, \mathrm{c}$ ). To assess the contribution of the force field to filopodia properties, freshly plated U2OS cells expressing MYO10-GFP were treated with either DMSO or the myosin II inhibitor blebbistatin for $1 \mathrm{~h}$ (Figure 5d). Interestingly, cells treated with blebbistatin displayed more, longer and curvier filopodia compared to DMSO-treated cells (Figure 5d,e). Altogether these data suggest that cellular contractility, transmitted to the ECM at focal adhesion, may contribute to the straightening of filopodia as well as restricting filopodia extension.

Discussion. We propose a simplified protocol and imaging strategy, relying on FBSR, which improves TFM measurements. Our strategy only requires off-the-shelf reagents and access to commonly available widefield or spinning-disk confocal microscopes and the analysis pipeline is fully compatible with freely available TFM analysis software. Importantly, FBSR-improved TFM data in combination with FBSR-enhanced detection of cellular proteins (e.g., paxillin or MYO10) can be used to correlate force data with specific cellular structures such as focal adhesions. In addition, we demonstrate that our workflow can be used to gain biologically relevant information and is suitable for fast and long-term live measurement of traction forces. Our strategy can also be used over a large field of view using low magnification high numerical aperture objectives.

One current limitation of the FBSR-based TFM workflow described here is that it is currently not compatible with $3 \mathrm{D}$ TFM (3D tracking of beads underneath cells plated on a 2D substrate) as demonstrated recently using SIM. ${ }^{4}$ However, the strategy described here has not yet reached full potential and could be further developed. In particular, as FBSR reconstruction algorithms are under constant development and continue to improve, ${ }^{19-21}$ we expect parallel advances in the quality of FBSR-TFM. For example, while FBSR algorithms can be capable of axial resolution improvement, ${ }^{19}$ this feature is not yet widely implemented and is likely to be improved in the future. In addition, as FBSR-TFM is fully compatible with existing TFM software, it can be further developed by fine-tuning the computational algorithms responsible for bead recognition, tracking and methods used to derive cellular forces. ${ }^{15,40,41}$ Here, we principally used the Fourier transform traction cytometry (FTTC) method to reconstruct forces, but it is tempting to speculate that even further quality enhancement could be gained by employing more computationally heavy mathematical frameworks. ${ }^{15}$ In addition, it is theoretically possible that the resolution of FBSR-TFM could be further enriched by mixing beads of different colors as demonstrated for confocal-based microscopy. ${ }^{12,22}$

Materials and Methods. Cells. U2OS and U-251 glioma cells were grown in DMEM/F-12 (Dulbecco's modified Eagle's medium/Nutrient Mixture F-12; Life Technologies, 10565018) supplemented with $10 \%$ fetal bovine serum (FCS) (Biowest, S1860). U2OS cells were purchased from DSMZ (Leibniz Institute DSMZ-German Collection of Microorganisms and Cell Cultures, Braunschweig DE, ACC 785). U-251 glioma cells were a generous gift from Professor David Odde (University of Minnesota, US). U2OS and U-251 glioma cells expressing endogenously tagged paxillin-GFP were generated using CRISPR/Cas9 as described by. ${ }^{42}$ The gRNA sequence targeting paxillin ( $5^{\prime}$-GCACCTAGCAGAAGAGCTTG- $3^{\prime}$ ) was cloned into the pSpCas9(BB)-2A-GFP backbone using the BbsI restriction site. ${ }^{43}$ Cells were then transfected with the GFP-Cas9-paxillin_gRNA construct and the template plasmid AICSDP-1:PXN-EGFP in an equimolar ratio (1:1). After transfection, cells were grown for 5 days before being sorted based on green fluorescence using a fluorescence-activated cell sorter (FACS; FACSAria IIu, BD). U2OS and U-251 glioma cells were transfected using Lipofectamine 3000 and the P3000TM Enhancer Reagent (Thermo Fisher Scientific) according to the manufacturer's instructions.

MCF10 DCIS.COM (DCIS.COM) lifeact-RFP cells were cultured in a 1:1 mix of DMEM (Sigma-Aldrich) and F12 (Sigma-Aldrich) supplemented with 5\% horse serum (16050122; GIBCO BRL), $20 \mathrm{ng} / \mathrm{mL}$ human EGF (E9644; SigmaAldrich), $0.5 \mathrm{mg} / \mathrm{mL}$ hydrocortisone (H0888-1G; SigmaAldrich), $100 \mathrm{ng} / \mathrm{mL}$ cholera toxin (C8052-1MG; SigmaAldrich), $10 \mu \mathrm{g} / \mathrm{mL}$ insulin (I9278-5 ML; Sigma-Aldrich), and $1 \%(\mathrm{v} / \mathrm{v})$ penicillin/streptomycin (P0781-100 ML; Sigma-Aldrich). Parental DCIS.COM cells were provided by J.F. Marshall (Barts Cancer Institute, Queen Mary University of London, London, England, UK). DCIS.COM lifeact-RFP cells were generated using lentiviruses, produced using $\mathrm{pCDH}$ LifeAct-mRFP, psPAX2, and pMD2.G constructs (see ref 44 for more details). To generate DCIS.COM organoids, DCIS.COM lifeact-RFP cells were seeded at very low density (500 cells per well) in low adhesion plates (Corning, 3474) for 
1 week before being transferred to TFM gels. The formation of organoids was monitored using bright field microscopy.

The human induced pluripotent stem cell (hPSC) line HEL24.3 was a kind gift from Professor Timo Otonkoski (University of Helsinki). This cell line was created using Sendai viruses ${ }^{45}$ and was cultured on Matrigel (Corning, 354277) in Essential 8 Basal medium (Life Technologies, A15169-01) supplemented with E8 supplements (Life Technologies, A1517-01). hPSC expressing endogenously tagged paxillin-GFP was described previously ${ }^{46}$ and were generated using CRISPR/Cas9 as described by. ${ }^{42}$

Antibodies and Plasmids. The mouse monoclonal antipaxillin antibody (PXN, Clone 349, 1:100 for IF) was provided by $\mathrm{BD}$ Biosciences (catalogue number: 610051). The mScarlet-MYO10 construct was described previously. ${ }^{28}$ The pmKate2-paxillin vector was purchased from Evrogen (cat.\# FP323). mEmerald-Paxillin-22 was a gift from Michael Davidson (Addgene plasmid \# 54219). ${ }^{47}$ psPAX2 and pMD2.G were gifts from D. Trono (École polytechnique fédérale de Lausanne, Lausanne, Switzerland; Addgene plasmid $\# 12260$ and \#12259). pCDH-LifeAct-mRFP was a gift from P. Caswell (University of Manchester, UK). AICSDP-1:PXNEGFP was a gift from The Allen Institute for Cell Science (Addgene plasmid \# 87420).

Sample Preparation for Light Microscopy. For SIM imaging, U2OS cells transiently expressing mEmeraldPaxillin-22 and Myosin-X-mScarlet were plated on high tolerance glass-bottom dishes (MatTek Corporation, coverslip \#1.7) precoated first with Poly-L lysine $(10 \mathrm{mg} / \mathrm{mL}, 1 \mathrm{~h}$ at 37 $\left.{ }^{\circ} \mathrm{C}\right)$ and then with bovine plasma fibronectin $(10 \mathrm{mg} / \mathrm{mL}, 2 \mathrm{~h}$ at $37{ }^{\circ} \mathrm{C}$ ). After $2 \mathrm{~h}$, samples were fixed and permeabilised simultaneously using a solution of $4 \%$ (wt/vol) PFA and $0.25 \%$ (v/v) Triton X-100 for $10 \mathrm{~min}$. Cells were then washed with PBS, quenched using a solution of $1 \mathrm{M}$ glycine for $30 \mathrm{~min}$, and incubated with SiR-actin (100 nM in PBS; Cytoskeleton; catalogue number: $\mathrm{CY}-\mathrm{SC} 001)$ at $4{ }^{\circ} \mathrm{C}$ until imaging (minimum length of staining, overnight at $4{ }^{\circ} \mathrm{C}$; maximum length, 1 week). Just before imaging, samples were washed three times in PBS and mounted in Vectashield (Vectorlabs).

For the filopodia formation assays, cells expressing human Myosin-X-GFP were plated on fibronectin-coated glass-bottom dishes (MatTek Corporation) for $1 \mathrm{~h}$ before being treated with either DMSO or $10 \mu \mathrm{M}$ blebbistatin for $1 \mathrm{~h}$. Samples were fixed for $10 \mathrm{~min}$ using a solution of $4 \%$ (wt/vol) PFA, then permeabilized using a solution of $0.25 \%(\mathrm{v} / \mathrm{v})$ Triton X-100 for $3 \mathrm{~min}$. Cells were then washed with PBS, quenched using a solution of $1 \mathrm{M}$ glycine for $30 \mathrm{~min}$, and incubated with the primary antibody for $1 \mathrm{~h}$ (1:100). After three washes, cells were incubated with a secondary antibody for $1 \mathrm{~h}$ (1:100). Samples were then washed three times and stored in PBS or in PBS containing SiR-actin (100 nM; Cytoskeleton; catalogue number: CY-SC001) at $4{ }^{\circ} \mathrm{C}$ until imaging. Just before imaging, samples were washed three times in PBS. Images were acquired using an spinning disk confocal microscope (100X objective). The number of filopodia per cell and their length was manually scored using Fiji. Filopodia curvature was analyzed, from manually traced filopodia, using the Kappa Fiji plug-in. $^{48}$

TFM Gel Preparation. The $35 \mathrm{~mm}$ glass-bottom dishes (Cellvis, D35-14-1N) were cleaned twice using absolute ethanol and air-dried. Dishes were treated with a Bind-Silane solution [714 $\mu \mathrm{l}$ Bind-SIlane (GE Healthcare, Silane A-174), $714 \mu \mathrm{l}$ Acetic acid and $8572 \mu \mathrm{l}$ of absolute ethanol) for $15 \mathrm{~min}$ ar RT. Dishes were then washed once with $95 \% \mathrm{EtOH}$ and twice with $\mathrm{mQH}_{2} \mathrm{O}$ before being left to dry completely. In parallel, $13 \mathrm{~mm}$ glass coverslips were coated with Poly-D-lysine $\left(10 \mu \mathrm{g} / \mathrm{mL}\right.$ in $\mathrm{mQH}_{2} \mathrm{O}$, Sigma-Aldrich, A-003-E) for $20 \mathrm{~min}$ at $+4{ }^{\circ} \mathrm{C}$, washed in $\mathrm{mQH}_{2} \mathrm{O}$ and then left to dry out. Fluorescent beads (either dark red, excitation $660 \mathrm{~nm} /$ emission $680 \mathrm{~nm}$; or orange, excitation $540 \mathrm{~nm} /$ emission $560 \mathrm{~nm}$; Thermo Fisher Scientific, F10720) were diluted 1:5000 in $\mathrm{mQH}_{2} \mathrm{O}$. The bead solution was then subjected to repeated sonication for $30 \mathrm{~s}$, followed by a 30-s pause, over a $10 \mathrm{~min}$ period. Importantly, beads were kept on ice for this entire duration to prevent bead clustering. Each Poly-D-lysine coated coverslip was then incubated with a $150 \mu \mathrm{L}$ drop of the bead solution at $+4{ }^{\circ} \mathrm{C}$ for $20 \mathrm{~min}$. Coverslips were then washed and kept in $\mathrm{mQH}_{2} \mathrm{O}$. Before use, the glass coverslips were left to dry completely.

A premixture composed of $40 \%$ acrylamide (Sigma-Aldrich, A4058), 2\% N, N'-Methylenebis(acrylamide) solution (SigmaAldrich, M1533) and PBS was prepared according to the desired gel stiffness (see Table 2). In this study, most

Table 2. TFM Gel Premixture Recipe

$\begin{array}{lcccccr}\text { AA. } & \text { Bis. AA. } & \text { PBS } & \begin{array}{l}\text { 200 nm beads } \\ (\mu \mathrm{L}) \text {, optional }\end{array} & \begin{array}{c}\text { APS. } \\ (\mu \mathrm{L})\end{array} & \begin{array}{c}\text { TEMED } \\ (\mu \mathrm{L})\end{array} & \begin{array}{c}\sim E \\ (\mathrm{kPa})\end{array} \\ 94 & 15 & 346 & 3.4 & 5 & 1 & 2.6 \\ 94 & 50 & 356 & 3.4 & 5 & 1 & 9.6 \\ 225 & 100 & 175 & 3.4 & 5 & 1 & 31.7\end{array}$

experiments were performed using $\sim 9.6 \mathrm{kPa}$ TFM gels with the exception of hPSC live imaging $(\sim 32 \mathrm{kPa}$ gel; Supplementary Figure $6 \mathrm{~b}$ ) and the large field of view TFM ( $\sim 2.6 \mathrm{kPa}$ and $\sim 9.6 \mathrm{kPa}$ gels, Figure $4 \mathrm{e}$ and $4 \mathrm{f})$.

From this stage onward, the premixture was kept on ice and sonicated for $30 \mathrm{~s}$ followed by a 30 -s pause over $10 \mathrm{~min}$. The premixture was then vortexed briefly, and 0.2\% TEMED (vol/ vol; Sigma-Aldrich, T9281), and 1\% ammonium persulfate (vol/vol; $10 \%$ stock solution) was added to start PAA polymerization. After a brief vortex, $11.8 \mu \mathrm{L}$ of gel mixture was added onto the glass of each glass-bottom dish. The beadcoated $13 \mathrm{~mm}$ coverslip was then carefully placed on top of the drop, ensuring that a thin layer of liquid remained between the two glass surfaces. The plates were then incubated dry at room temperature for $60 \mathrm{~min}$ after which they were submerged in PBS to allow the careful removal of the top glass coverslip using forceps. Gels can be stored submerged in PBS at $+4{ }^{\circ} \mathrm{C}$ for up to a week.

In the experiments where spinning-disk confocal TFM was performed in addition to FBSR TFM, $200 \mathrm{~nm}$ green fluorescent beads (excitation $505 \mathrm{~nm} /$ emission $515 \mathrm{~nm}$ ) (Life Technologies, F8811) were also added to the premixture prior to the sonication step (Table 2).

To allow for functionalization, TFM gels were incubated for $30 \mathrm{~min}$ at RT with an activation solution $[0.2 \mathrm{mg} / \mathrm{mL}$ of SulfoSANPAH (Thermo Fisher Scientific, 22589), 2 mg/mL N-(3(dimethylamino)propyl)- $N^{\prime}$-ethylcarbodiimide hydrochloride (EDC) (Sigma-Aldrich, 03450) diluted in $50 \mathrm{mM}$ HEPES (4-(2-hydroxyethyl)-1-piperazineethanesulfonic acid; SigmaAldrich, H0887)] under gentle agitation. ${ }^{49}$ The glass-bottom dishes containing the gels were then irradiated, without their plastic lids, with ultraviolet (UV) light for $10 \mathrm{~min}$ using a UVchamber (Jelight Company Inc., UVO CLEANER, 342-220). Gels were then washed three times with PBS and coated with 
either $10 \mu \mathrm{g} / \mathrm{mL}$ fibronectin (Merck-Millipore, 341631) (U2OS, DCIS.COM, and U-251 cells) or $5 \mu \mathrm{g} / \mathrm{mL}$ vitronectin (Thermo Fisher Scientific, A14700) (hPSCs).

Microscopy Setup. The spinning disk confocal microscope (spinning-disk confocal) used was a Marianas spinning disk imaging system with a Yokogawa CSU-W1 scanning unit on an inverted Zeiss Axio Observer Z1 microscope controlled by SlideBook 6 (Intelligent Imaging Innovations, Inc.). Images were acquired using a Photometrics Evolve, back-illuminated EMCCD camera $(512 \times 512$ pixels $)$. The microscope was used either in confocal or widefield mode as indicated in the figure legends. The objectives used were a $20 \times$ (NA 0.8 air, Plan Apochromat, DIC) objective (Zeiss), a $63 \times$ (NA 1.15 water, LD C-Apochromat) objective (Zeiss), and a 100× (NA 1.4 oil, Plan-Apochromat, M27) objective.

The structured illumination microscope (SIM) used was DeltaVision OMX v4 (GE Healthcare Life Sciences) fitted with a 60x Plan-Apochromat objective lens, 1.42 NA (immersion oil RI of 1.516) used in SIM illumination mode (five phases $\times$ three rotations). Emitted light was collected on a front-illuminated pco.edge sCMOS (pixel size $6.5 \mathrm{~mm}$, readout speed $95 \mathrm{MHz}$; PCO AG) controlled by SoftWorx.

Traction Force Microscopy. Cells were plated on TFM gels (in a $1 \mathrm{~mL}$ volume of media) and left to adhere for at least $2 \mathrm{~h}$ prior to imaging. To avoid drifting during imaging, the spinning disk microscope was prewarmed to $37{ }^{\circ} \mathrm{C}$ prior to image acquisition. To perform TFM measurements, beads were imaged before (Pre) and after (Post) removing cells, the Pre and Post images were then aligned (see methods below), the beads detected in both images and their movements tracked and local forces measured (see methods below). To perform FBSR, 100 frames of the Pre and Post bead planes and of the paxillin staining (when indicated) were acquired. In between acquiring the Pre and Post images, the cells were removed by adding $500 \mu \mathrm{L}$ of a $20 \%$ SDS in $\mathrm{mQH}_{2} \mathrm{O}(5 \mathrm{~min}$ incubation).

To perform the blebbistatin treatment experiment (Figure $3 c, d)$, a first set of FBSR images of beads and focal adhesions were acquired (first Pre image). Then a warm solution containing $10 \mu \mathrm{M}$ blebbistatin (final concentration in cell medium; Stemcell Technologies, 72402) or DMSO (SigmaAldrich, D2650) was added to the cells (1 mL added) for 15 min. A second set of FBSR images of beads and focal adhesions was then acquired (second Pre image). The cells were then detached as described above and the final set of FBSR images was then acquired (Post image). To perform extended live TFM imaging of the iPSCs and U-251 cells, FBSR image sets of the beads were acquired every $5 \mathrm{~min}$ and the cells were detached as described above. When estimating the temporal resolution of FBSR TFM, DCIS.COM cells were seeded on 9.6 $\mathrm{kPa}$ fibronectin-coated gel and imaged continuously over 16 min. To perform the experiments using a large field of view and 20x air objective the cells (U2OS or DCIS.COM) were seeded on fibronectin-coated PAA hydrogels with $200 \mathrm{~nm}$ beads cast inside as in the classical TFM protocol. When using the U2OS cells the stiffness of the hydrogel was $2.6 \mathrm{kPa}(\sim 3 \mathrm{~h})$ whereas the DCIS.com organoids were seeded on top of 9.6 $\mathrm{kPa}$ gels $(\sim 24 \mathrm{~h})$. One-hundred pre and post images were taken as described previously, however, using $20 \times$ air objective. U2OS cells were imaged using the widefield mode of the spinning disk confocal microscope whereas the DCIS.COM organoids were imaged using confocal mode. Only the beads were imaged using 100 frame acquisition.
Images of SiR-DNA and Lifeact were acquired only once per time frame.

Image Alignment. Prior to bead tracking and force mapping, the pre and post bead images were aligned in the Fiji distribution of Image ${ }^{50-52}$ using either the NanoJ-Core ${ }^{53}$ or the "Linear stack alignment with SIFT" plugins. The "Linear stack alignment with SIFT" plugin was only used when affine registration was required. In all cases, the first Pre image was used as a reference image. For the blebbistatin and for the live TFM experiments, focal adhesion images were registered with bead images using the drift table generated by the NanoJ-Core plugin. ${ }^{53}$

Generation of Simulated Bead Images. The positions of the beads were randomly distributed over the chosen field-ofview size (here $128 \times 128 \mu \mathrm{m}^{2}$ ). The number of beads was chosen to obtain the desired density. Each bead was simulated as a group of $\sim 650$ dyes homogeneously distributed in a $40 \mathrm{~nm}$ sphere. The field of simulated dye distribution thus obtained was simulated over a $5 \mathrm{~nm}$ resolution grid. Each dye was allowed to blink independently with on/off rate of $100 \mathrm{~s}^{-1}$ and $50 \mathrm{~s}^{-1}$ respectively over the entire acquisition without bleaching (200 frames at $10 \mathrm{~ms}$ exposure). The simulated fluorescence image produced by this distribution of beads was created by convolution with a Gaussian kernel with $\sigma=0.21 \times \frac{\lambda}{N A},{ }^{54}$ where $\lambda$ is the wavelength of emission (here $700 \mathrm{~nm}$ ) and $N A$ is the numerical aperture of the microscope (here $N A=1.12$ ). A pixel size of $250 \mathrm{~nm}$ was chosen for the final fluorescence image in agreement with our experimental setup. A realistic Poisson photon noise and a Gaussian read-out noise were added to the images to simulate experimental data set $(S N R \approx 7)$. Subsequently, a displacement field previously obtained from a representative experimental data set was applied to the original bead positions and the fluorescence simulation was run again independently. This way, a pair of stacks can be generated from the same bead distribution and density. Simulated stacks were then processed and analyzed as described for the experimentally generated images.

FBSR Processing. FBSR processing was performed using NanoJ-LiveSRRF and SACD. ${ }^{21}$ NanoJ-LiveSRRF is the newest implementation of NanoJ-SRRF within the ImageJ software. NanoJ-LiveSRRF is available upon request (will be openly available for download soon), whereas NanoJ-SRRF is already an open-source software. ${ }^{20}$

For FBSR processing using LiveSRRF, 100 frames were used for the reconstruction. The parameter sweep option as well as SQUIRREL analyses (resolution-scaled error (RSE) and resolution-scaled Pearson (RSP) values), ${ }^{27}$ integrated within LiveSRRF, were used to define the optimal reconstruction parameters (32 different conditions as shown in Supplementary Figure 1b). RSE and RSP are two metrics that indicate how well the two images agree at the resolution of the widefield image. In SQUIRREL, the super-resolution images are blurred to achieve an equivalent resolution as that of the widefield image and the two are then compared. Any deviation between the wide-field image and the resolution-scaled superresolution image will highlight artifacts/agreement. RSE and RSP respectively represent the sum of all the intensity errors between the two images (for RSE the smaller the number, the better the agreement, 0 being a perfect agreement) and a correlation-based metric (based on Pearson correlation, therefore for RSP, the closer to 1, the better the agreement). 
Table 3. LiveSRRF Parameters

\begin{tabular}{|c|c|c|c|c|c|c|c|}
\hline & $\begin{array}{l}\text { vibration } \\
\text { correction }\end{array}$ & radius & sensitivity & magnification & $\begin{array}{l}\text { temporal } \\
\text { analysis }\end{array}$ & $\begin{array}{l}\text { intensity } \\
\text { weighting }\end{array}$ & $\begin{array}{l}\text { macro-pixel patterning } \\
\text { correction }\end{array}$ \\
\hline $40 \mathrm{~nm}$ beads images taken with confocal & on & 1.5 & 1 & 5 & average & on & on \\
\hline $40 \mathrm{~nm}$ beads images taken with widefield & on & 2 & 2 & 5 & average & on & on \\
\hline $\begin{array}{l}200 \mathrm{~nm} \text { beads images acquired with } 20 \times \text { air } \\
\text { objective and widefield }\end{array}$ & on & 2 & 2 & 5 & average & on & on \\
\hline Paxillin images & on & 2 & 1 & 5 & average & on & on \\
\hline MYO10 images & on & 2 & 2 & 5 & average & on & on \\
\hline Lifeact images & on & 2 & 1 & 5 & average & on & on \\
\hline
\end{tabular}

Table 4. Key Parameters for TFM Analyses Using MATLAB-Based Software

\begin{tabular}{|c|c|c|c|}
\hline & bead detection parameters & $\begin{array}{l}\text { "template size" and "maximum displacement for } \\
\text { calculating displacement field" }\end{array}$ & force field calculation \\
\hline Figure $2 c$ & $\begin{array}{l}\text { high-resolution subsampling of beads and use subpixel } \\
\text { correlation via image interpolation }\end{array}$ & 20 and $21 \mathrm{px}$ & $\begin{array}{l}\text { FTTC (Fourier transform } \\
\text { traction cytometry) }\end{array}$ \\
\hline Figure $3 b$ & $\begin{array}{l}\text { high-resolution subsampling of beads and use subpixel } \\
\text { correlation via image interpolation }\end{array}$ & 40 and $41 \mathrm{px}$ & FTTC \\
\hline Figure $3 c, d$ & $\begin{array}{l}\text { high-resolution subsampling of beads and use subpixel } \\
\text { correlation via image interpolation }\end{array}$ & 80 and $81 \mathrm{px}$ & FTTC \\
\hline Figure 4a & PIV & 80 and $81 \mathrm{px}$ & FTTC \\
\hline Figure $4 b$ & $\begin{array}{l}\text { high-resolution subsampling of beads and use subpixel } \\
\text { correlation via image interpolation }\end{array}$ & 40 and $41 \mathrm{px}$ & FTTC \\
\hline Figure $4 c$ & $\begin{array}{l}\text { high-resolution subsampling of beads and use subpixel } \\
\text { correlation via image interpolation }\end{array}$ & 80 and $81 \mathrm{px}$ & FTTC \\
\hline Figure $4 \mathrm{e}$ and $\mathrm{f}$ & $\begin{array}{l}\text { high-resolution subsampling of beads and use subpixel } \\
\text { correlation via image interpolation }\end{array}$ & 20 and $21 \mathrm{px}$ & FTTC \\
\hline Figure $5 b$ & $\begin{array}{l}\text { high-resolution subsampling of beads and use subpixel } \\
\text { correlation via image interpolation }\end{array}$ & 60 and $61 \mathrm{px}$ & FTTC \\
\hline
\end{tabular}

For each liveSRRF setting combination, RSP and RSE values, and bead density were measured and ranked. The optimal LiveSRRF settings were then determined based on all criteria (best overall rank) (see Supplementary Figure 2c) and the final parameters used to process images are listed in Table 3.

For FBSR processing with $S A C D,{ }^{21}$ the first 50 frames were used for the reconstruction. SACD reconstructions were performed within MATLAB (Mathworks, version R2019a) and the following parameters: $A, 1.15$; pixel size, $247 \times 10^{-9}$; lambda, $647 \times 10^{-9}$; iter, 1 ; mag, 5; square, 2 , order, 3 . The MATLAB script used to process SACD images is available on GitHub and can be found at https://github.com/ guijacquemet/.

Quantification of Bead Density. Bead density was quantified by dividing the number of beads detected (in a field of view) by the size of the field of view. The number of beads for each field of view was measured in Fiji using the "find maxima" option. The threshold used was tuned for each data set so that only beads were counted. Importantly, the number of beads measured using this strategy was nearly identical to the number of beads identified by the MATLAB-based TFM software.

Assessment of Image Resolution. Fourier ring correlation (FRC) analysis was performed using NanoJ-SQUIRREL implemented within Image J. ${ }^{27}$ As FRC analyses require two images to be performed, raw FBSR data sets (composed of 100 frames) were split in half by sorting the odd and even frames. Even and odd data sets were then processed separately as indicated (Average $\mathrm{Z}$ projection, LiveSRRF or SACD) and the two output images were used for the FRC analyses.

Image decorrelation analysis was performed in Image J using the Image decorrelation analysis plugin. ${ }^{55}$ This analysis requires a single image as input and therefore the full FBSR data sets were used here.

Bead Tracking and Local Force Measurements. The bead tracking and local force measurements were performed either using MATLAB (Mathworks, version R2019a) or using Fiji. ${ }^{50-52}$ For the MATLAB-based analyses, the TFM software developed by the Danuser laboratory was used. ${ }^{15}$ If not indicated otherwise, bead trackings were performed by crosscorrelation within the search window. Key parameters used can be found in Table 4.

To generate the displacement and traction maps in Fiji, the particle image velocity (PIV) plugin and the Fourier transform traction cytometry (FTTC) plugin ${ }^{32}$ were used. The aligned images of the pre and post TFM images of the beads were first processed with the PIV plugin using the correlation coefficient iteration option (interrogation window sizes: 128 pixel first round, 64 pixel second round and 32 pixel third round). The resulting PIV text file was then saved and plotted as a displacement map using the plot function. Images shown in Supplementary Figure $5 \mathrm{~d}$ were postprocessed using the normalized median test option (parameters used: 0.2 for noise and 5.0 for threshold). The traction force maps were generated using the ImageJ FTTC plugin (Poisson ratio, 0.5; Young's modulus, $10 \mathrm{kPa}$; regularization parameter, $4.0 \times$ $10^{-10}$ ). The total forces were calculated by measuring the integrated density of the 32-bit images produced by the plugin.

Filopodia and Force Field Alignment. U2OS cells transiently expressing mEmerald-Paxillin-22 and mScarletMYO10 were seeded on $9.6 \mathrm{kPa}$ fibronectin-coated TFM gels for at least $3 \mathrm{~h}$. The $40 \mathrm{~nm}$ beads, MYO10, and paxillin were all imaged to allow for FBSR processing using Live-SRRF (100 frames). TFM analyses were then performed using the MATLAB software as previously described. To measure the filopodia alignment to the force field, images containing the 
force field vectors as well as the MYO10 and paxillin staining were generated in MATLAB. The angle between the filopodia and the force field was measured in ImageJ using the angle calculation tool. The closest force field vector to each filopodia tip was used.

Image Denoising Using Noise2VOID. The signal-tonoise ratios of endogenously tagged paxillin (Figure 4a) and of DNA (SiR-DNA) (Figure 4c) were improved using the recent denoising approach Noise2VOID, ${ }^{34}$ which is based on convolutional neural networks. Noise2VOID was executed through the Google Colaboratory platform, which can run Jupyter Notebooks in the cloud. The Jupyter Notebooks used are available on GitHub and can be found at https://github. com/guijacquemet/.

Statistical Analysis. Dot plots and box plots were generated using the online tool PlotsOfData (https:// huygens.science.uva.nl/PlotsOfData/). ${ }^{56}$ Correlation analyses were performed using Spearman's Rank-order. Statistical analyses were performed using the Mann-Whitney $U$ test or a randomization test as indicated in the figure legends. Randomization tests were performed using the online tool PlotsOfDifferences (https://huygens.science.uva.nl/ PlotsOfDifferences/). ${ }^{57}$ The error bars presented in figures depict the standard deviation. $\mathrm{N}$ numbers are indicated in the figure legends.

\section{ASSOCIATED CONTENT}

\section{(s) Supporting Information}

The Supporting Information is available free of charge at https://pubs.acs.org/doi/10.1021/acs.nanolett.9b04083.

Video of Live FBSR TFM in a migrating glioma cell (MP4)

Video of Live FBSR TFM in a DCIS.COM cell (MP4)

Results of simulations that demonstrate that FBSR TFM improves TFM measurements; bead detection within TFM gels using FBSR; full field of view of Figure 2; data presented in Figure 2 reanalyzed using PIV; full field of view of Figure 3; examples of live cell imaging fluctuation-based TFM; effect of exposure time and number of frames in SRRF reconstructions (PDF)

\section{AUTHOR INFORMATION}

\section{Corresponding Authors}

Guillaume Jacquemet - Turku Bioscience Centre, University of Turku and Åbo Akademi University, FI-20520 Turku, Finland; Faculty of Science and Engineering, Cell Biology, Åbo Akademi University, 20520 Turku, Finland;

Email: guillaume.jacquemet@abo.fi

Johanna Ivaska - Turku Bioscience Centre, University of Turku and Åbo Akademi University, FI-20520 Turku, Finland; Department of Biochemistry, University of Turku, FIN-20520

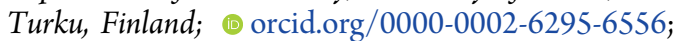
Email: johanna.ivaska@utu.fi

\section{Authors}

Aki Stubb - Turku Bioscience Centre, University of Turku and Åbo Akademi University, FI-20520 Turku, Finland

Romain F. Laine - MRC-Laboratory for Molecular Cell Biology, University College London, London WC1E 6BT, U.K.; The Francis Crick Institute, London NW1 1AT, U.K.; (1) orcid.org/0000-0002-2151-4487
Mitro Miihkinen - Turku Bioscience Centre, University of Turku and Åbo Akademi University, FI-20520 Turku, Finland

Hellyeh Hamidi - Turku Bioscience Centre, University of Turku and Åbo Akademi University, FI-20520 Turku, Finland

Camilo Guzmán - Turku Bioscience Centre, University of Turku and Åbo Akademi University, FI-20520 Turku, Finland

Ricardo Henriques - MRC-Laboratory for Molecular Cell Biology, University College London, London WC1E 6BT, U.K.; The Francis Crick Institute, London NW1 1AT, U.K.

Complete contact information is available at:

https://pubs.acs.org/10.1021/acs.nanolett.9b04083

\section{Author Contributions}

Conceptualization, A.S., G.J., and J.I.; methodology, A.S., R.L., and G.J.; formal analysis, G.J., R.L., M.M., C.G., and A.S.; investigation, A.S. and M.M.; resources, J.I., R.L., and R.H.; writing original draft, A.S., G.J., and J.I.; writing review and editing, A.S., R.L., H.H., R.H., G.J., and J.I.; visualization, A.S., H.H., G.J., and J.I.; supervision, G.J. and J.I.; funding acquisition, G.J. and J.I.

\section{Notes}

The authors declare no competing financial interest.

The authors declare that the data supporting the findings of this study are available within the article and from the authors upon request.

\section{ACKNOWLEDGMENTS}

This study has been supported by the Academy of Finland (G.J., and J.I.), the Academy of Finland CoE for Translational Cancer Research (J.I.), an ERC CoG grant (\#615258), and the Sigrid Juselius Foundation (J.I and G.J.) and the Finnish Cancer Organization (J.I.). G.J. was supported by grants awarded by the Åbo Akademi University Research Foundation (CoE CellMech) and by the Drug Discovery and Diagnostics strategic funding of Åbo Akademi University. A.S. has been supported by the University of Turku Doctoral programme for Molecular Medicine (TuDMM). R.F.L. and R.H. were funded by grants from the UK Biotechnology and Biological Sciences Research Council [BB/S507532/1], the UK Medical Research Council $[\mathrm{MR} / \mathrm{K} 015826 / 1]$, the Wellcome Trust [203276/Z/ $16 / Z]$, and core funding by the MRC Laboratory for Molecular Cell Biology, University College London [MC_UU12018/7]. We thank Aleksi Isomursu for providing us with the U-251 glioma cell line expressing endogenously tagged paxillin-GFP. We thank J. Siivonen and P. Laasola for technical assistance and M. Saari for help with the microscopes. The Cell Imaging and Cytometry Core facility (Turku Bioscience, University of Turku, Åbo Akademi University and Biocenter Finland) is acknowledged for services, instrumentation, and expertise. The Allen Institute for Cell Science, David Odde (University of Minnesota, US), Patrick Caswell (University of Manchester), Michael Davidson, and Timo Otonkoski (University of Helsinki) are acknowledged for providing reagents.

\section{REFERENCES}

(1) Conway, J. R. W.; Jacquemet, G. Cell Matrix Adhesion in Cell Migration. Essays Biochem. 2019, 63, No. EBC20190012.

(2) Kechagia, J. Z.; Ivaska, J.; Roca-Cusachs, P. Integrins as Biomechanical Sensors of the Microenvironment. Nat. Rev. Mol. Cell Biol. 2019, 20 (8), 457-473.

(3) Heisenberg, C.-P.; Bellaïche, Y. Forces in Tissue Morphogenesis and Patterning. Cell 2013, 153 (5), 948-962. 
(4) Wickström, S. A.; Niessen, C. M. Cell Adhesion and Mechanics as Drivers of Tissue Organization and Differentiation: Local Cues for Large Scale Organization. Curr. Opin. Cell Biol. 2018, 54, 89-97.

(5) Duscher, D.; Maan, Z. N.; Wong, V. W.; Rennert, R. C.; Januszyk, M.; Rodrigues, M.; Hu, M.; Whitmore, A. J.; Whittam, A. J.; Longaker, M. T.; et al. Mechanotransduction and Fibrosis. J. Biomech. 2014, 47 (9), 1997-2005.

(6) Segel, M.; Neumann, B.; Hill, M. F. E.; Weber, I. P.; Viscomi, C.; Zhao, C.; Young, A.; Agley, C. C.; Thompson, A. J.; Gonzalez, G. A.; et al. Niche Stiffness Underlies the Ageing of Central Nervous System Progenitor Cells. Nature 2019, 573 (7772), 130-134.

(7) Broders-Bondon, F.; Ho-Bouldoires, T. H. N.; FernandezSanchez, M.-E.; Farge, E. Mechanotransduction in Tumor Progression: The Dark Side of the Force. J. Cell Biol. 2018, 217 (5), 1571-1587.

(8) Lampi, M. C.; Reinhart-King, C. A. Targeting Extracellular Matrix Stiffness to Attenuate Disease: From Molecular Mechanisms to Clinical Trials. Sci. Transl. Med. 2018, 10 (422), eaao0475.

(9) Roca-Cusachs, P.; Conte, V.; Trepat, X. Quantifying Forces in Cell Biology. Nat. Cell Biol. 2017, 19 (7), 742-751.

(10) Style, R. W.; Boltyanskiy, R.; German, G. K.; Hyland, C.; MacMinn, C. W.; Mertz, A. F.; Wilen, L. A.; Xu, Y.; Dufresne, E. R. Traction Force Microscopy in Physics and Biology. Soft Matter 2014, 10 (23), 4047-4055.

(11) Saxena, M.; Changede, R.; Hone, J.; Wolfenson, H.; Sheetz, M. P. Force-Induced Calpain Cleavage of Talin Is Critical for Growth, Adhesion Development, and Rigidity Sensing. Nano Lett. 2017, 17 (12), 7242-7251.

(12) Plotnikov, S. V.; Sabass, B.; Schwarz, U. S.; Waterman, C. M. Chapter 20 - High-Resolution Traction Force Microscopy. In Methods in Cell Biology; Waters, J. C., Wittman, T., Eds.; Academic Press, 2014; Vol. 123, pp 367-394. DOI: 10.1016/B978-0-12-420138-5.00020-3. (13) Colin-York, H.; Shrestha, D.; Felce, J. H.; Waithe, D.; Moeendarbary, E.; Davis, S. J.; Eggeling, C.; Fritzsche, M. SuperResolved Traction Force Microscopy (STFM). Nano Lett. 2016, 16 (4), 2633-2638.

(14) Colin-York, H.; Javanmardi, Y.; Barbieri, L.; Li, D.; Korobchevskaya, K.; Guo, Y.; Hall, C.; Taylor, A.; Khuon, S.; Sheridan, G. K.; et al. Spatiotemporally Super-Resolved Volumetric Traction Force Microscopy. Nano Lett. 2019, 19 (7), 4427-4434.

(15) Han, S. J.; Oak, Y.; Groisman, A.; Danuser, G. Traction Microscopy to Identify Force Modulation in Subresolution Adhesions. Nat. Methods 2015, 12 (7), 653-656.

(16) Zündel, M.; Ehret, A. E.; Mazza, E. Factors Influencing the Determination of Cell Traction Forces. PLoS One 2017, 12 (2), e0172927.

(17) Bagshaw, C. R.; Cherny, D. Blinking Fluorophores: What Do They Tell Us about Protein Dynamics? Biochem. Soc. Trans. 2006, 34 (5), 979-982.

(18) van de Linde, S.; Sauer, M. How to Switch a Fluorophore: From Undesired Blinking to Controlled Photoswitching. Chem. Soc. Rev. 2014, 43 (4), 1076-1087.

(19) Dertinger, T.; Colyer, R.; Iyer, G.; Weiss, S.; Enderlein, J. Fast, Background-Free, 3D Super-Resolution Optical Fluctuation Imaging (SOFI). Proc. Natl. Acad. Sci. U. S. A. 2009, 106 (52), 22287-22292.

(20) Gustafsson, N.; Culley, S.; Ashdown, G.; Owen, D. M.; Pereira, P. M.; Henriques, R. Fast Live-Cell Conventional Fluorophore Nanoscopy with ImageJ through Super-Resolution Radial Fluctuations. Nat. Commun. 2016, 7, 12471.

(21) Zhao, W.; Liu, J.; Kong, C.; Zhao, Y.; Guo, C.; Liu, C.; Ding, X.; Ding, X.; Tan, J.; Li, H. Faster Super-Resolution Imaging with Auto-Correlation Two-Step Deconvolution. ArXiv180907410 [physics.optics] 2018.

(22) Plotnikov, S. V.; Pasapera, A. M.; Sabass, B.; Waterman, C. M. Force Fluctuations within Focal Adhesions Mediate ECM-Rigidity Sensing to Guide Directed Cell Migration. Cell 2012, 151 (7), 15131527.
(23) Culley, S.; Tosheva, K. L.; Matos Pereira, P.; Henriques, R. SRRF: Universal Live-Cell Super-Resolution Microscopy. Int. J. Biochem. Cell Biol. 2018, 101, 74-79.

(24) Knoll, S. G.; Ali, M. Y.; Saif, M. T. A. A Novel Method for Localizing Reporter Fluorescent Beads near the Cell Culture Surface for Traction Force Microscopy. J. Visualized Exp. 2014, 91, 51873.

(25) Gutierrez, E.; Tkachenko, E.; Besser, A.; Sundd, P.; Ley, K.; Danuser, G.; Ginsberg, M. H.; Groisman, A. High Refractive Index Silicone Gels for Simultaneous Total Internal Reflection Fluorescence and Traction Force Microscopy of Adherent Cells. PLoS One 2011, 6 (9), No. e23807.

(26) Lendenmann, T.; Schneider, T.; Dumas, J.; Tarini, M.; Giampietro, C.; Bajpai, A.; Chen, W.; Gerber, J.; Poulikakos, D.; Ferrari, A.; et al. Cellogram: On-the-Fly Traction Force Microscopy. Nano Lett. 2019, 19 (10), 6742-6750.

(27) Culley, S.; Albrecht, D.; Jacobs, C.; Pereira, P. M.; Leterrier, C.; Mercer, J.; Henriques, R. Quantitative Mapping and Minimization of Super-Resolution Optical Imaging Artifacts. Nat. Methods 2018, 15 (4), 263-266.

(28) Jacquemet, G.; Stubb, A.; Saup, R.; Miihkinen, M.; Kremneva, E.; Hamidi, H.; Ivaska, J. Filopodome Mapping Identifies P130Cas as a Mechanosensitive Regulator of Filopodia Stability. Curr. Biol. 2019, 29 (2), 202-216.e7.

(29) Närvä, E.; Stubb, A.; Guzmán, C.; Blomqvist, M.; Balboa, D.; Lerche, M.; Saari, M.; Otonkoski, T.; Ivaska, J. A Strong Contractile Actin Fence and Large Adhesions Direct Human Pluripotent Colony Morphology and Adhesion. Stem Cell Rep. 2017, 9 (1), 67-76.

(30) Georgiadou, M.; Lilja, J.; Jacquemet, G.; Guzmán, C.; Rafaeva, M.; Alibert, C.; Yan, Y.; Sahgal, P.; Lerche, M.; Manneville, J.-B.; et al. AMPK Negatively Regulates Tensin-Dependent Integrin Activity. J. Cell Biol. 2017, 216 (4), 1107-1121.

(31) Hakanpaa, L.; Kiss, E. A.; Jacquemet, G.; Miinalainen, I.; Lerche, M.; Guzmán, C.; Mervaala, E.; Eklund, L.; Ivaska, J.; Saharinen, P. Targeting B1-Integrin Inhibits Vascular Leakage in Endotoxemia. Proc. Natl. Acad. Sci. U. S. A. 2018, 115 (28), E6467E6476.

(32) Tseng, Q.; Duchemin-Pelletier, E.; Deshiere, A.; Balland, M.; Guillou, H.; Filhol, O.; Théry, M. Spatial Organization of the Extracellular Matrix Regulates Cell-Cell Junction Positioning. Proc. Natl. Acad. Sci. U. S. A. 2012, 109, 201106377.

(33) Kurzawa, L.; Vianay, B.; Senger, F.; Vignaud, T.; Blanchoin, L.; Théry, M. Dissipation of Contractile Forces: The Missing Piece in Cell Mechanics. Mol. Biol. Cell 2017, 28 (14), 1825-1832.

(34) Krull, A.; Buchholz, T.-O.; Jug, F. Noise2Void - Learning Denoising from Single Noisy Images. ArXiv181110980 [cs.CV] 2018.

(35) Sunyer, R.; Conte, V.; Escribano, J.; Elosegui-Artola, A.; Labernadie, A.; Valon, L.; Navajas, D.; García-Aznar, J. M.; Muñoz, J. J.; Roca-Cusachs, P.; et al. Collective Cell Durotaxis Emerges from Long-Range Intercellular Force Transmission. Science 2016, 353 (6304), 1157-1161.

(36) Yoshie, H.; Koushki, N.; Kaviani, R.; Tabatabaei, M.; Rajendran, K.; Dang, Q.; Husain, A.; Yao, S.; Li, C.; Sullivan, J. K.; et al. Traction Force Screening Enabled by Compliant PDMS Elastomers. Biophys. J. 2018, 114 (9), 2194-2199.

(37) Jacquemet, G.; Hamidi, H.; Ivaska, J. Filopodia in Cell Adhesion, 3D Migration and Cancer Cell Invasion. Curr. Opin. Cell Biol. 2015, 36, 23-31.

(38) Jacquemet, G.; Baghirov, H.; Georgiadou, M.; Sihto, H.; Peuhu, E.; Cettour-Janet, P.; He, T.; Perälä, M.; Kronqvist, P.; Joensuu, H.; et al. L-Type Calcium Channels Regulate Filopodia Stability and Cancer Cell Invasion Downstream of Integrin Signalling. Nat. Commun. 2016, 7, 13297.

(39) Alieva, N. O.; Efremov, A. K.; Hu, S.; Oh, D.; Chen, Z.; Natarajan, M.; Ong, H. T.; Jégou, A.; Romet-Lemonne, G.; Groves, J. T.; et al. Myosin IIA and Formin Dependent Mechanosensitivity of Filopodia Adhesion. Nat. Commun. 2019, 10 (1), 3593.

(40) Holenstein, C. N.; Silvan, U.; Snedeker, J. G. High-Resolution Traction Force Microscopy on Small Focal Adhesions - Improved 
Accuracy through Optimal Marker Distribution and Optical Flow

Tracking. Sci. Rep. 2017, 7 (1), 1-14.

(41) Huang, Y.; Schell, C.; Huber, T. B.; Şimşek, A. N.; Hersch, N.; Merkel, R.; Gompper, G.; Sabass, B. Traction Force Microscopy with Optimized Regularization and Automated Bayesian Parameter Selection for Comparing Cells. Sci. Rep. 2019, 9 (1), 1-16.

(42) Roberts, B.; Haupt, A.; Tucker, A.; Grancharova, T.; Arakaki, J.; Fuqua, M. A.; Nelson, A.; Hookway, C.; Ludmann, S. A.; Mueller, I. A.; et al. Systematic Gene Tagging Using CRISPR/Cas9 in Human Stem Cells to Illuminate Cell Organization. Mol. Biol. Cell 2017, 28 (21), 2854-2874.

(43) Roberts, B.; Haupt, A.; Tucker, A.; Grancharova, T.; Arakaki, J.; Fuqua, M. A.; Nelson, A.; Hookway, C.; Ludmann, S. A.; Mueller, I. A.; et al. Systematic Gene Tagging Using CRISPR/Cas9 in Human Stem Cells to Illuminate Cell Organization. Mol. Biol. Cell 2017, 28 (21), 2854-2874.

(44) Jacquemet, G.; Paatero, I.; Carisey, A. F.; Padzik, A.; Orange, J. S.; Hamidi, H.; Ivaska, J. FiloQuant Reveals Increased Filopodia Density during Breast Cancer Progression. J. Cell Biol. 2017, 216, 3387.

(45) Trokovic, R.; Weltner, J.; Noisa, P.; Raivio, T.; Otonkoski, T. Combined Negative Effect of Donor Age and Time in Culture on the Reprogramming Efficiency into Induced Pluripotent Stem Cells. Stem Cell Res. 2015, 15 (1), 254-262.

(46) Stubb, A.; Guzmán, C.; Närvä, E.; Aaron, J.; Chew, T.-L.; Saari, M.; Miihkinen, M.; Jacquemet, G.; Ivaska, J. Superresolution Architecture of Cornerstone Focal Adhesions in Human Pluripotent Stem Cells. Nat. Commun. 2019, 10 (1), 1-15.

(47) Paszek, M. J.; DuFort, C. C.; Rubashkin, M. G.; Davidson, M. W.; Thorn, K. S.; Liphardt, J. T.; Weaver, V. M. Scanning Angle Interference Microscopy Reveals Cell Dynamics at the Nanoscale. Nat. Methods 2012, 9 (8), 825-827.

(48) Mary, H.; Brouhard, G. J. Kappa $(\kappa)$ : Analysis of Curvature in Biological Image Data using B-splines. bioRxiv 2019, DOI: 10.1101/ 852772v1.full.

(49) Lerche, M.; Elosegui-Artola, A.; Kechagia, J. Z.; Guzmán, C.; Georgiadou, M.; Gullberg, D.; Roca-Cusachs, P.; Peuhu, E.; Ivaska, J. Integrin Binding Dynamics Modulate Ligand-Specific Mechanosensing in Mammary Gland Fibroblasts. bioRxiv 2019, 570721.

(50) Schindelin, J.; Arganda-Carreras, I.; Frise, E.; Kaynig, V.; Longair, M.; Pietzsch, T.; Preibisch, S.; Rueden, C.; Saalfeld, S.; Schmid, B.; et al. Fiji: An Open-Source Platform for Biological-Image Analysis. Nat. Methods 2012, 9 (7), 676-682.

(51) Rueden, C. T.; Schindelin, J.; Hiner, M. C.; DeZonia, B. E.; Walter, A. E.; Arena, E. T.; Eliceiri, K. W. ImageJ2: ImageJ for the next Generation of Scientific Image Data. BMC Bioinf. 2017, 18 (1), 529.

(52) Schneider, C. A.; Rasband, W. S.; Eliceiri, K. W. NIH Image to ImageJ: 25 Years of Image Analysis. Nat. Methods 2012, 9, 671-675.

(53) Laine, R. F.; Tosheva, K. L.; Gustafsson, N.; Gray, R. D. M.; Almada, P.; Albrecht, D.; Risa, G. T.; Hurtig, F.; as, A.-C. L.; Baum, B.; et al. NanoJ: A High-Performance Open-Source Super-Resolution Microscopy Toolbox. J. Phys. D: Appl. Phys. 2019, 52 (16), 163001.

(54) Zhang, B.; Zerubia, J.; Olivo-Marin, J.-C. Gaussian Approximations of Fluorescence Microscope Point-Spread Function Models. Appl. Opt. 2007, 46 (10), 1819-1829.

(55) Descloux, A.; Grußmayer, K. S.; Radenovic, A. Parameter-Free Image Resolution Estimation Based on Decorrelation Analysis. Nat. Methods 2019, 16 (9), 918-924.

(56) Postma, M.; Goedhart, J. PlotsOfData-A Web App for Visualizing Data Together with Their Summaries. PLoS Biol. 2019, 17 (3), No. e3000202.

(57) Goedhart, J. PlotsOfDifferences - a Web App for the Quantitative Comparison of Unpaired Data. bioRxiv 2019, 578575. 